\title{
On the correspondence between surface operators in Argyres-Douglas theories and modules of chiral algebra
}

\author{
Takahiro Nishinaka, ${ }^{a}$ Shinya Sasa ${ }^{b}$ and Rui-Dong $\mathbf{Z h u}^{b}$ \\ ${ }^{a}$ Department of Physical Sciences, College of Science and Engineering, Ritsumeikan University, \\ Shiga 525-8577, Japan \\ ${ }^{b}$ Department of Physics, The University of Tokyo, \\ Bunkyo-ku, Tokyo 113-8654, Japan \\ E-mail: nishinak@fc.ritsumei.ac.jp, sasa@hep-th.phys.s.u-tokyo.ac.jp, \\ nick_zrd@hep-th.phys.s.u-tokyo.ac.jp
}

Abstract: We compute the Schur index of Argyres-Douglas theories of type $\left(A_{N-1}, A_{M-1}\right)$ with surface operators inserted, via the Higgsing prescription proposed by D. Gaiotto, L. Rastelli and S.S. Razamat. These surface operators are obtained by turning on positiondependent vacuum expectation values of operators in a UV theory which can flow to the Argyres-Douglas theories. We focus on two series of $\left(A_{N-1}, A_{M-1}\right)$ theories; one with $\operatorname{gcd}(N, M)=1$ and the other with $M=N(k-1)$ for an integer $k \geq 2$. Our results are identified with the characters of non-vacuum modules of the associated $2 \mathrm{~d}$ chiral algebras, which explicitly confirms a remarkable correspondence recently discovered by C. Cordova, D. Gaiotto and S.-H. Shao.

KeYwords: Conformal and W Symmetry, Duality in Gauge Field Theories, Conformal Field Theory, Supersymmetric Gauge Theory

ARXIV EPRINT: 1811.11772 


\section{Contents}

1 Introduction 1

2 TQFT description and Higgsing prescription 4

2.1 TQFT description for Schur index 4

$\begin{array}{ll}2.2 & \text { Higgsing prescription }\end{array}$

$3\left(A_{N-1}, A_{M-1}\right)$ theories with $\operatorname{gcd}(N, M)=1 \quad 9$

3.1 Modules in $\mathcal{W}_{N}$ minimal models $\quad 9$

$\begin{array}{lll}3.2 \text { Review of }\left(A_{1}, A_{2 k}\right) \text { series } & 10\end{array}$

$3.3\left(A_{N-1}, A_{M-1}\right)$ series with $\operatorname{gcd}(N, M)=1 \quad 11$

$4 \quad\left(A_{N-1}, A_{N(k-1)-1}\right)$ theories $\quad 13$

4.1 Logarithmic $\mathcal{B}(k)_{A_{N-1}}$ algebra $\quad 13$

$\begin{array}{lll}4.2 & \text { Schur indices with surface operator insertions } & 14\end{array}$

$\begin{array}{ll}4.3 \text { Comparison to the characters of } \mathcal{B}(k)_{A_{N-1}} \text { modules } & 16\end{array}$

$\begin{array}{lll}5 & \text { Conclusion and discussion } & 17\end{array}$

$\begin{array}{lr}\text { A Explicit } \boldsymbol{q} \text {-series for lower-rank examples } & \mathbf{1 8}\end{array}$

$\begin{array}{ll}\text { A.1 }\left(A_{2}, A_{n-1}\right) \text { series with } \operatorname{gcd}(3, n)=1 & 19\end{array}$

A.2 $\left(A_{3}, A_{4}\right)$ theory and $\left(A_{4}, A_{5}\right)$ theory 22

B Generalized Euler identities $\quad 24$

\section{Introduction}

The $4 \mathrm{~d} / 2 \mathrm{~d}$ duality, established after the groundbreaking work of [1-4], is remarkable progress in the study of $4 \mathrm{~d} \mathcal{N}=2$ field theories. This states that, for a large class of $4 \mathrm{~d} \mathcal{N}=2$ theories called class $\mathcal{S}$ theories [5, 6], physical quantities in $4 \mathrm{~d}$ theories are encoded in a two-dimensional theory on a punctured Riemann surface, $\mathcal{C}$. This duality is naturally understood when we recall that class $\mathcal{S}$ theories are constructed by compactifying a $6 \mathrm{~d} \mathcal{N}=(2,0)$ theory (with "gauge algebra" $\mathfrak{g}$ ) on $\mathcal{C} .{ }^{1}$ The relevant $2 \mathrm{~d}$ theory on $\mathcal{C}$ depends on the $4 \mathrm{~d}$ physical quantity we consider. In particular, when we consider the Schur index of a $4 \mathrm{~d} \mathcal{N}=2$ superconformal field theory (SCFT) of class $\mathcal{S}$,

$$
\mathcal{I}(q ; \mathbf{x}) \equiv \operatorname{Tr}_{\mathcal{H}}(-1)^{F} q^{E-R} \prod_{\ell=1}^{\operatorname{rank} G_{F}} x_{\ell}^{f_{\ell}}
$$

\footnotetext{
${ }^{1}$ To keep the $4 \mathrm{~d} \mathcal{N}=2$ supersymmetry, a partial topological twist is needed on $\mathcal{C}$.
} 
the relevant $2 \mathrm{~d}$ theory is a topological quantum field theory (TQFT) called the $q$-deformed Yang-Mills theory on $\mathcal{C}$. Indeed, the Schur index of class $\mathcal{S}$ theories were shown to be identical to the correlation function of the $q$-deformed Yang-Mills theory on $\mathcal{C}[3,4]$ (See also [7] for a review). In the above definition of the Schur index, $\mathcal{H}$ is the Hilbert space of local operators, $E$ and $R$ are respectively the scaling dimension and the $\mathrm{SU}(2)_{R}$ charge, $G_{F}$ is the flavor symmetry group, and $f_{\ell}$ are the flavor charges. ${ }^{2}$ In this paper we encounter cases with $G_{F}=\mathrm{SU}(N)$, in which case we take $f_{k}$ to be minus the $k$-th Cartan generator of SU $(N)$. The BPS operators giving non-vanishing contributions to the Schur index are called "Schur operators".

In the above discussion, the $2 \mathrm{~d}$ theory is a TQFT and therefore its correlation function is independent of the complex structure of $\mathcal{C}$. This reflects the fact that the $4 \mathrm{~d}$ superconformal index is independent of marginal couplings [8]. Indeed, the complex structure deformations of $\mathcal{C}$ correspond to marginal deformations of the $4 \mathrm{~d}$ theory [5]. ${ }^{3}$ On the other hand, each puncture on $\mathcal{C}$ corresponds to an external state of the $2 \mathrm{~d}$ TQFT, which is naturally associated with a wavefunction of the form,

$$
f_{R}^{(i)}\left(q ; \mathbf{x}^{(i)}\right)
$$

Here $i=1, \cdots, m$ is the label of the puncture, $R$ stands for certain irreducible representation of $\mathfrak{g}$, and $\mathbf{x}^{(i)} \equiv\left\{x_{k}^{(i)}\right\}$ are fugacity parameters associated to the flavor symmetry introduced by the puncture. ${ }^{4}$ Suppose that $\mathcal{C}$ is a Riemann surface of genus $g$ with $m$ punctures. Then the correlation function of the $2 \mathrm{~d}$ TQFT is given by

$$
\mathcal{I}(q ; \mathbf{x})=\sum_{R}\left(C_{R}(q)\right)^{2 g-2+m} \prod_{i=1}^{m} f_{R}^{(i)}\left(q ; x_{k}^{(i)}\right),
$$

where $C_{R}(q)$ is the structure constant of the TQFT. The $4 \mathrm{~d} / 2 \mathrm{~d}$ duality implies that this is identical to the Schur index (1.1) of the corresponding 4d SCFT.

The Schur index also plays an important role in another $4 \mathrm{~d} / 2 \mathrm{~d}$ correspondence discovered in [15]. This correspondence states that, for any $4 \mathrm{~d} \mathcal{N}=2$ SCFT (even without class $\mathcal{S}$ description), the space of local operators contributing to the Schur index is equipped with the structure of a chiral algebra, or equivalently, a vertex operator algebra (VOA). In this context, the Schur index is identified with the character of the vacuum module of the chiral algebra. ${ }^{5}$ Furthermore, it has recently been shown that the Schur indices in the presence of a large class of surface operators are identified with the characters of

\footnotetext{
${ }^{2}$ We call the global symmetry that commutes with the $4 \mathrm{~d} \mathcal{N}=2$ superconformal symmetry "flavor symmetry".

${ }^{3}$ Note that an $\mathcal{N}=2$ marginal coupling in theories of class $\mathcal{S}$ SCFTs are not necessarily encoded in the complex structure of $\mathcal{C}$. For examples of marginal coupling arising from a puncture on $\mathcal{C}$, see [9-13]. A relation between the space of marginal couplings (i.e., conformal manifold) and the associated chiral algebra was studied in [14].

${ }^{4}$ Note that the $\left\{\mathbf{x}^{(1)}, \cdots, \mathbf{x}^{(m)}\right\}$ can be reorganized into $\mathbf{x} \equiv\left\{x_{1}, \cdots, x_{\mathrm{rank} G_{F}}\right\}$.

${ }^{5}$ For remarkable unitary/non-unitary relations in this context, see [16]. Note also that the associated chiral algebra is defined in a two-dimensional plane inside the four-dimensional spacetime. Therefore this $4 \mathrm{~d} / 2 \mathrm{~d}$ correspondence is different from the AGT correspondence.
} 
some non-vacuum modules of the chiral algebra [17, 18]. ${ }^{6}$ In particular in [18], the Schur indices of free hypermultiplets and the $\left(A_{1}, A_{3}\right),\left(A_{1}, A_{5}\right)$ and $\left(A_{1}, A_{2 n}\right)$ Argyres-Douglas (AD) theories [20-25] for all positive integers $n$, with surface operator insertions, were computed and shown to be identical to the characters of irreducible modules of the associated chiral algebras.

In this article, we use the TQFT description to compute the Schur indices with surface operator insertions for two infinite series of AD theories, generalizing the careful studies in [18]. The first series is the $\left(A_{N-1}, A_{M-1}\right)$ theories for integers $N, M \geq 2$ satisfying $\operatorname{gcd}(N, M)=1$, whose chiral algebra was conjectured in [26] to be the vacuum sector of the $\mathcal{W}_{N}$ minimal model labeled by $(N, N+M) .{ }^{7}$ The second series is the $\left(A_{N-1}, A_{N(k-1)-1}\right)$ theories for integers $N, k \geq 2,{ }^{8}$ whose chiral algebra was conjectured in [29-32] based on the closed-form formula for the Schur indices (without surface operators) obtained in [33, 34]. ${ }^{9}$ Note that the first series for $N=2$ and the second series for $N=2$ and $k=3,4$ were already studied in [18]. Since the structure of the non-vacuum modules is generally very complicated, generalizing them to the whole theories in the two series is highly non-trivial. For the $\left(A_{N-1}, A_{M-1}\right)$ theories with $\operatorname{gcd}(M, N)=1$, we show that the Schur indices with surface operator insertions reproduce all and only the characters of the irreducible modules of the $(N, M+N) \mathcal{W}_{N}$ minimal model. For the $\left(A_{N-1}, A_{N(k-1)-1}\right)$ theories, we show a similar statement for all $k \geq 2$ with $N=2$. For $N>2$, we give a natural conjecture on modules of the logarithmic $\mathcal{B}(k)_{A_{N-1}}$ algebra [31].

To evaluate the Schur index in the presence of surface operators, we adopt the prescription proposed in [42]. This prescription first introduces a UV SCFT of class $\mathcal{S}$ whose Riemann surface $\mathcal{C}$ is obtained by adding a regular puncture to the original Riemann surface. We then Higgs the flavor symmetry associated with the added regular puncture, which triggers an RG-flow going back to the original SCFT. The main point of the prescription is that, by turning on a position-dependent vacuum expectation value (VEV) of the Higgs branch operator in this RG-flow, we can introduce surface operators in the IR 4d SCFT. From the index viewpoint, this operation corresponds to taking a particular limit of the flavor fugacity so that the UV index is divergent. This divergence reflects the fact that the there are decoupled massless Nambu-Goldstone (NG) multiplets associated with the symmetry breaking. The IR index with the surface operator inserted is then obtained by removing the contributions of these decoupled NG multiplets from the UV index.

This article is organized as follows. We provide brief reviews on the TQFT description and the Higgsing prescription for the Schur index in section 2. In section 3, we study the Schur indices of the $\left(A_{N-1}, A_{M-1}\right)$ theories with surface operator insertions for $M$ and $N$ such that $\operatorname{gcd}(M, N)=1$. We particularly find that all and only the characters of irreducible modules of the $(N, M+N) \mathcal{W}_{N}$ minimal model are reproduced from the

\footnotetext{
${ }^{6}$ It was also shown in [19] that, for some Argyres-Douglas theories, the Schur index in the presence of line defects are also related to the characters of non-vacuum modules. Its relation to the surface operator insertions is discussed in [18].

${ }^{7}$ For an interesting observation on the ODE/IM correspondence for these theories, see [27, 28].

${ }^{8}$ To be precise, the case of $N=k=2$ is not an AD theory but the theory of a free hypermultiplet.

${ }^{9}$ These chiral algebras are deeply related to recent progress in the study of VOAs appearing in logarithmic CFTs [35-39]. For the Macdonald index and the small $S^{1}$-reduction of the $\left(A_{1}, A_{2 k-3}\right)$ theories, see [40, 41].
} 
Higgsing. In section 4, we study the Schur indices with surface operator insertions for the $\left(A_{N-1}, A_{N(k-1)-1}\right)$ theory. For $N=2$, we find that the Schur indices coincide with the characters of modules of the logarithmic $B_{k}$ algebra [29], by generalizing the check performed in [18].

\section{TQFT description and Higgsing prescription}

We here give a brief review of the Schur index of $4 \mathrm{~d} \mathcal{N}=2$ SCFTs with and without surface operator insertions. In particular, we review in section 2.1 the TQFT description of the Schur index of theories of class $\mathcal{S}$ [3]. In section 2.2, we review that the index in the presence of a surface operator is obtained via the so-called "Higgsing prescription" [42].

\subsection{TQFT description for Schur index}

Let us first review how to compute the Schur index of the $\left(A_{N-1}, A_{M-1}\right)$ theories from the TQFT picture. These theories are of class $\mathcal{S}[5,6]$, and therefore associated with a $6 \mathrm{~d}$ "gauge algebra" $\mathfrak{g}$ and a punctured Riemann surface $\mathcal{C} .{ }^{10}$ As discussed in [25], the $\left(A_{N-1}, A_{M-1}\right)$ theories are realized by $\mathfrak{g}=A_{N-1}$ and $\mathcal{C}$ being a sphere with an irregular puncture. Therefore we focus on $\mathfrak{g}=A_{N-1}$ in this paper. To each puncture on $\mathcal{C}$, we assign a wavefunction $f_{R}^{(i)}\left(q, \mathbf{x}^{(i)}\right)$ according to the type of the puncture, where we recall that $R$ is an irreducible representation of $A_{N-1}$, and $\mathbf{x}^{(i)}=\left(x_{1}^{(i)}, \cdots, x_{N-1}^{(i)}\right)$ stands for the fugacities corresponding to the flavor symmetry associated with the $i$-th puncture. The Schur index of the theory is then written as (1.3), where $g$ is the genus of the Riemann surface, and $m$ is the number of punctures. When $\mathcal{C}$ involves an irregular puncture, $g$ must be zero for the $\mathrm{U}(1)_{R}$ symmetry of the $4 \mathrm{~d}$ theory to be preserved [25]. The factor $C_{R}$ is given by

$$
\left(C_{R}\right)^{-1}=\frac{\chi_{R}^{\mathrm{SU}(N)}\left(q^{\rho}\right)}{\prod_{i=2}^{N}\left(q^{i} ; q\right)}
$$

where $\chi_{R}^{\mathrm{SU}(N)}(x)$ is the character of the $\mathrm{SU}(N)$ representation $R$, and $\rho=\frac{1}{2} \sum_{\alpha \in \Delta^{+}} \alpha$ is the Weyl vector of $\mathrm{SU}(N) .{ }^{11}$ We use the notation such that $\chi_{R}^{\mathrm{SU}(N)}(x)=\sum_{\mu \in P} m_{\lambda}(\mu) x^{\mu}$, where $\lambda$ is the highest weight of $R, P$ is the weight lattice of $\mathrm{SU}(N), m_{\lambda}(\mu)$ is the multiplicity of $\mu$ in the representation $R$, and $x^{\mu}$ is a formal exponential such that $x^{\mu_{1}} x^{\mu_{2}}=x^{\mu_{1}+\mu_{2}}$. In substituting $x=q^{\rho}$ in $\chi_{R}^{\mathrm{SU}(N)}(x)$, we interpret $\left(q^{\rho}\right)^{\mu}$ to mean $q^{(\rho, \mu)}$.

As mentioned above, the expression for the wave function depends on the type of the puncture. The wavefunction for a full regular puncture [5] is given by

$$
f_{R}^{\mathrm{full}}(q, \mathbf{x})=\frac{\chi_{R}^{\mathrm{SU}(N)}(\mathbf{x})}{(q ; q)^{N-1} \prod_{\alpha \in \Delta}\left(q x^{\alpha} ; q\right)},
$$

\footnotetext{
${ }^{10}$ While these theories have class $\mathcal{S}$ constructions, they were originally constructed in [23] by using type IIB string theory on Calabi-Yau singularities.

${ }^{11}$ Here $\Delta_{+}$is the set of positive roots.
} 
where $\Delta$ is the set of roots of $\mathfrak{g}=A_{N-1}$, and $\chi_{R}^{\mathrm{SU}(N)}(\mathbf{x}) \equiv \chi_{R}^{\mathrm{SU}(N)}(x)$ with the identifications $x^{-\omega_{i}}=x_{i}$ for fundamental weights $\omega_{i}$. Especially the wavefunction for $\mathfrak{g}=A_{1}$ is given by

$$
f_{R}^{\mathrm{full}}(q, x)=\frac{\chi_{R}^{\mathrm{SU}(2)}(x)}{(q ; q)\left(q x^{2} ; q\right)\left(q x^{-2} ; q\right)},
$$

with $\chi_{R}^{\mathrm{SU}(2)}(x)=\left(x^{\operatorname{dim} R}-x^{-\operatorname{dim} R}\right) /\left(x-x^{-1}\right)$, where we write $x_{1}$ as $x$ for simplicity.

Since the Riemann surface $\mathcal{C}$ for $\mathrm{AD}$ theories involves an irregular puncture [24, 25], we also need an expression for the wavefunction of the irregular puncture [33, 34, 43]. Indeed, the $\left(A_{N-1}, A_{M-1}\right)$ theory is realized by $\mathcal{C}$ being a sphere with one irregular puncture. Therefore its Schur index is written as

$$
\mathcal{I}_{\left(A_{N-1}, A_{M-1}\right)}(q, \mathbf{x})=\sum_{R}\left(C_{R}\right)^{-1} \widetilde{f}_{R}^{\left(A_{N-1}, A_{M-1}\right)}(q ; \mathbf{x}),
$$

where $R$ runs over all irreducible representations of $A_{N-1}$, and $\widetilde{f}_{R}^{\left(A_{N-1}, A_{M-1}\right)}$ stands for the wave function for the irregular puncture. For the $\left(A_{N-1}, A_{M-1}\right)$ theory with $\operatorname{gcd}(N, M)=1$, the expression for $\widetilde{f}_{R}$ is simplified since no flavor symmetry arises from the puncture. Indeed, it is conjectured in [43] to be given by ${ }^{12}$

$$
\begin{aligned}
\tilde{f}_{R}^{\left(A_{N-1}, A_{M-1}\right)}(q)= & \oint\left(\prod_{k=1}^{N-1} \frac{d z_{k}}{2 \pi i z_{k}}\right) \Delta(\mathbf{z}) P . E \cdot\left[-\frac{q^{N+M}}{1-q^{N+M}} \chi_{a d j}^{\mathrm{SU}(N)}(\mathbf{z})\right] \chi_{R}(\mathbf{z}) \\
& (\text { if } \operatorname{gcd}(M, N)=1)
\end{aligned}
$$

where $\Delta(\mathbf{z})=\frac{1}{N !} \prod_{1 \leq i \leq j \leq N-1}\left(1-z^{\alpha_{i}+\cdots+\alpha_{j}}\right)\left(1-z^{-\left(\alpha_{i}+\cdots+\alpha_{j}\right)}\right)$, and P.E. $\left[g\left(q, z_{i}\right)\right] \equiv$ $\exp \left(\sum_{k=1}^{\infty} \frac{g\left(q^{k}, z_{i}^{k}\right)}{k}\right)$ for any function $g$ of the fugacities. For $N=2$ and $M=2 n+1$, this expression reduces to

$$
\widetilde{f}_{R}^{\left(A_{1}, A_{2 n}\right)}(q)=\left\{\begin{array}{cc}
(-1)^{m} q^{m(m+1)\left(n+\frac{3}{2}\right)} \operatorname{dim} R=2 m+1: \text { odd } \\
0 & \text { otherwise }
\end{array}\right.
$$

On the other hand, for $\left(A_{N-1}, A_{M-1}\right)$ theories with $\operatorname{gcd}(N, M) \neq 1$, the irregular puncture is associated with a non-trivial flavor symmetry. In particular, when $M=N(k-1)$ for an integer $k \geq 2$, the irregular puncture is associated with a $\mathrm{U}(1)^{N-1}$ flavor symmetry. The expression for the wave function in this case was conjectured in [34] as $^{13}$

$$
\widetilde{f}_{R}^{\left(A_{N-1}, A_{N(k-1)-1}\right)}(q, \mathbf{x})=\prod_{j=1}^{\infty}\left(1-q^{j}\right)^{1-N} q^{k C_{2}(R)} \sum_{\mu \in R} q^{-\frac{k}{2} F^{i j} \mu_{i} \mu_{j}} x^{\mu},
$$

where $\mu$ runs over all weights belonging to the representation $R,\left(\mu_{1}, \cdots, \mu_{N-1}\right)$ are the Dynkin labels of $\mu$, and $x^{\mu}$ is again the formal exponential such that $x^{\mu} x^{\nu}=x^{\mu+\nu}$ and

\footnotetext{
${ }^{12}$ Note here that there is no flavor fugacity $\mathbf{x}$ is associated with the irregular puncture in the case of $\operatorname{gcd}(M, N)=1$.

${ }^{13}$ For $\left(A_{1}, A_{2 k-3}\right)$ theories, this wave function was first conjectured in [33].
} 
$x^{-\omega_{i}}=x_{i}$ for the fundamental weights $w_{i} \cdot{ }^{14}$ The factor $C_{2}(R)$ is the quadratic Casimir invariant, and $\left(F^{i j}\right)$ is the inverse Cartan matrix:

$$
\left(F^{i j}\right)=\frac{1}{N}\left(\begin{array}{ccccc}
N-1 & N-2 & N-3 & \ldots & 1 \\
N-2 & 2(N-2) & 2(N-3) & \ldots & 2 \\
N-3 & 2(N-3) & 3(N-3) & \ldots & 3 \\
\vdots & \vdots & \vdots & \ddots & \vdots \\
1 & 2 & 3 & \ldots & N-1
\end{array}\right)
$$

In particular, for $N=2$, this expression reduces to

$$
\tilde{f}_{R}^{\left(A_{1}, A_{2 k-3}\right)}(q ; x)=\frac{q^{k \frac{(\mathrm{dim} R)^{2}-1}{4}}}{(q ; q)} \operatorname{tr}_{R}\left[x^{2 J_{3}} q^{-k\left(J_{3}\right)^{2}}\right],
$$

where $J_{3}$ is the Cartan of $A_{1}$ such that the fundamental representation has eigenvalues $J_{3}= \pm \frac{1}{2}$.

\subsection{Higgsing prescription}

In [42], a prescription to evaluate the Schur index in the presence of a class of surface operators was proposed, which we call the "Higgsing prescription". ${ }^{15}$ We here review and describe it in a more general setup so that we can apply it to the $\left(A_{N-1}, A_{M-1}\right)$ theories in the following sections. Suppose that we are interested in a class $\mathcal{S}$ theory associated with the Riemann surface $\mathcal{C}_{\mathrm{IR}}$. To introduce a surface operator in it, we first consider a UV $\mathcal{N}=2$ SCFT associated with a Riemann surface $\mathcal{C}_{\text {UV }}$ which is obtained by adding an extra regular puncture to $\mathcal{C}_{\mathrm{IR}}$. The original SCFT is recovered by removing the regular puncture from $\mathcal{C}_{\mathrm{UV}}$, which corresponds to giving a non-vanishing VEV to the Higgs branch operator associated with the regular puncture. The non-vanishing VEV triggers an RG-flow from the UV to the IR SCFT. As discussed in [42], the existence of this RG-flow implies that the index of the IR theory is obtained by taking a limit of the UV index. ${ }^{16}$

To describe this limit, let us focus on the case in which the additional regular puncture on $\mathcal{C}_{\mathrm{UV}}$ is a full puncture and therefore associated with an $\mathrm{SU}(N)$ flavor symmetry. ${ }^{17}$ The original discussion of [42] focuses on Higgsing the simple regular puncture, whose generalization to the full regular puncture is straightforward but needs a little care about the decoupled Nambu-Goldstone multiplets. See discussions around eq. (2.44) and in section 4.3 of [52] for this generalization without surface operator insertions. The existence of the extra full puncture on $\mathcal{C}_{\mathrm{UV}}$ implies that the UV SCFT has an additional flavor $\operatorname{SU}(N)$ current. In the superconformal multiplet containing the flavor current, there exists a scalar Schur operator called a "flavor moment map" in the adjoint representation of the flavor symmetry. We denote by $\mathcal{O}_{\alpha_{\ell}}$ the flavor moment map associated with the $\ell$-th simple root,

\footnotetext{
${ }^{14}$ Note that this choice of $x_{i}$ is slightly different from the one in [34].

${ }^{15}$ See [44-50] for earlier applications of this prescription.

${ }^{16}$ For another interesting class of RG-flows from $\mathrm{AD}$ theories of class $\mathcal{S}$, see [51].

${ }^{17}$ While this Higgsing is possible for any type of regular puncture, we will only use the full puncture in this paper.
} 
$\alpha_{\ell}$, of $\operatorname{SU}(N)$. The contribution from $\mathcal{O}_{\alpha_{\ell}}$ to the factor $\prod_{k=1}^{N-1} y_{k}^{f_{k}}$ in the Schur index (1.1) is $y^{\alpha_{1}} \cdot{ }^{18}$ This and the fact that every flavor moment map has $E=2 R=1$ implies that $\mathcal{O}_{\alpha_{\ell}}$ contributes $q y^{\alpha_{\ell}}$ to the Schur index.

To remove the full puncture from the Riemann surface, we need to completely Higgs the flavor $\mathrm{SU}(N)$ symmetry. This can be achieved by giving a non-vanishing VEV to $\mathcal{O}_{\alpha_{\ell}}$ for all $\ell=1, \cdots, N-1 .{ }^{19}$ Let us first focus on $\mathcal{O}_{\alpha_{1}}$ associated with the first simple root. According to [42], giving a non-vanishing VEV to $\mathcal{O}_{\alpha_{1}}$ triggers an RG-flow whose IR fixed-point has the following Schur index

$$
\mathcal{I}_{\mathrm{vec}}(q) \cdot \lim _{y_{1} \rightarrow y_{1}^{*}}\left(\left(1-q y^{\alpha_{1}}\right) \mathcal{I}_{\mathrm{UV}}(q ; \mathbf{x}, \mathbf{y})\right),
$$

where $\mathcal{I}_{\text {vec }}(q) \equiv(q ; q)^{2}$ is the Schur index of a free vector multiplet, and $y_{1}=y_{1}^{*}$ is the solution to the equation

$$
q y^{\alpha_{1}}=1
$$

Note that, since $y^{\alpha_{1}}=y_{1}^{-2} y_{2}$, the above equation can be solved for $y_{1}$. In the expression $(2.10)$, the factor $\left(1-q y^{\alpha_{1}}\right)$ removes the index contribution of operators $\left(\mathcal{O}_{\alpha_{1}}\right)^{n}$ for all $n \in \mathbb{N}$. Indeed, these operators induce a factor $1 /\left(1-q y^{\alpha_{1}}\right)$ in $\mathcal{I}_{\mathrm{UV}}(q ; \mathbf{x}, \mathbf{y})$, which is divergent in the limit $y_{1} \rightarrow y_{1}^{*}$. This divergence reflects the fact that there is a massless mode corresponding to the VEV of $\mathcal{O}_{\alpha_{1}}$. A remarkable observation of [42] is that this divergent factor, multiplied by $\mathcal{I}_{\mathrm{vec}}(q)^{-1}$, is the index contribution from the decoupled Nambu-Goldstone (NG) multiplet associated with the symmetry breaking. The factor $\mathcal{I}_{\text {vec }}(q)\left(1-q y^{\alpha_{1}}\right)$ precisely removes this index contribution, leaving the index of the IR theory without the decoupled NG sector.

While the index (2.10) still depends on $y_{2}, \cdots, y_{N-2}$ and $y_{N-1}$, turning on the VEVs of all $\mathcal{O}_{\alpha_{\ell}}$ leads to an index independent of $\mathbf{y}$, which is identified with the Schur index of the IR SCFT associated with the Riemann surface $\mathcal{C}_{\mathrm{IR}}$. In other words, the IR index is evaluated as

$$
\mathcal{I}_{\mathrm{IR}}(q ; \mathbf{x})=\left(\mathcal{I}_{\mathrm{vec}}(q)\right)^{\frac{N(N-1)}{2}} \cdot \lim _{\mathbf{y} \rightarrow \mathbf{y}^{*}}\left(\mathcal{I}_{\mathrm{UV}}(q ; \mathbf{x}, \mathbf{y}) \prod_{1 \leq i \leq j \leq N-1}\left(1-\prod_{\ell=i}^{j}\left(q y^{\alpha_{\ell}}\right)\right)\right),
$$

where $\mathbf{y}=\mathbf{y}^{*}$ is the simultaneous solution to the equations $q y^{\alpha_{\ell}}=1$ for $\ell=1, \cdots, N-1$, or equivalently

$$
\prod_{j=1}^{N-1}\left(y_{j}\right)^{c_{\ell j}}=q \quad \text { for } \quad \ell=1, \cdots, N-1
$$

where $\left(c_{i j}\right)$ is the Cartan matrix of $\mathrm{SU}(N)$. Note that turning on the VEV of all $\mathcal{O}_{\alpha_{\ell}}$ gives rise to $\frac{N(N-1)}{2}$ decoupled NG multiplets, corresponding to the positive roots of $\mathrm{SU}(N) .{ }^{20}$

\footnotetext{
${ }^{18}$ Here $y^{\lambda}$ is a formal exponential such that $y^{-\omega_{i}}=y_{i}$ for fundamental weights $\omega_{i}$. As mentioned in section 1 , we take the $k$-th flavor charge $f_{k}$ to be minus the $k$-th Cartan generator of $\mathrm{SU}(N)$.

${ }^{19}$ From the chiral algebra point of view, this corresponds to the quantum Drinfeld-Sokolov reduction associated with the principal embedding of $\mathfrak{s u}(2)$ in $\mathfrak{s u}(N)$.

${ }^{20}$ This can be seen explicitly in the wave function for the full puncture shown in (2.2).
} 
The factor $\left(\mathcal{I}_{\mathrm{vec}}(q)\right)^{\frac{N(N-1)}{2}} \prod_{1 \leq i \leq j \leq N-1}\left(1-\prod_{\ell=i}^{j}\left(q y^{\alpha \ell}\right)\right)$ then removes the index contribution from these decoupled NG multiplets. ${ }^{21}$

Note that the limit (2.10) can be regarded as taking the residue of the UV index at $y_{1}=y_{1}^{*}$. Indeed, this residue computation is the original form of the prescription proposed in [42], in which Higgsing flavor $\mathrm{U}(1)$ and $\mathrm{SU}(2)$ symmetries is mainly studied. On the other hand, the IR index (2.12) obtained by completely Higgsing the $\mathrm{SU}(N)$ flavor symmetry is not regarded as the residue of the UV index at $\mathbf{y}=\mathbf{y}^{*}$. Indeed, the residue is the coefficient of $\prod_{\ell=1}^{N-1}\left(1-q y^{\alpha_{\ell}}\right)^{-1}$ in the Laurent series around $\mathbf{y}=\mathbf{y}^{*}$ while $(2.12)$ is essentially the coefficient of $\prod_{1 \leq i \leq j \leq N-1}\left(1-\prod_{\ell=i}^{j} q y^{\alpha_{\ell}}\right)^{-1}$. This discrepancy arises since the spontaneously broken $\mathrm{SU}(N)$ symmetry implies $\frac{N(N-1)}{2}$ decoupled NG multiplets corresponding to the number of positive roots. This point was essentially noted in section 4.3 of [52].

Let us now turn to the Schur index with surface operator insertions. For $\mathcal{O}_{\alpha_{\ell}}$ and any positive integer $s_{\ell}$, the Schur index also has a contribution from the derivative operator $\left(\sigma_{+\dot{+}}^{\mu} \partial_{\mu}\right)^{s_{\ell}} \mathcal{O}_{\alpha_{\ell}}$. Since a single derivative increases the scaling dimension by one without changing the $\mathrm{SU}(2)_{R}$ and flavor charges, the index contribution from the derivative operator is $q^{1+s_{\ell}} y^{\alpha_{\ell}}$. The UV index then has a factor $1 /\left(1-q^{1+s_{\ell}} y^{\alpha_{\ell}}\right)$, which is divergent at $q^{1+s_{\ell}} y^{\alpha_{\ell}}=1$, or equivalently at

$$
\prod_{j=1}^{N-1}\left(y_{j}\right)^{c_{\ell j}}=q^{n_{\ell}} \quad \text { for } \quad \ell=1, \cdots, N-1
$$

where we defined $\mathbf{n}=\left(n_{1}, \cdots, n_{N-1}\right)$ for later use by $n_{\ell} \equiv 1+s_{\ell}$. Let us denote by $\mathbf{y}=\mathbf{y}_{\mathbf{n}}^{*}$ the simultaneous solution to the equations (2.14). An important observation of [42] is that the limit $\mathbf{y} \rightarrow \mathbf{y}_{\mathbf{n}}^{*}$ corresponds to turning on a position-dependent VEV of $\mathcal{O}_{\alpha_{\ell}}$ and therefore leads to the insertion of a surface operator labeled by $\mathbf{n}$ in the IR theory. We denote this surface operator by $\mathbb{S}^{\mathbf{n}}$. According to [42], the IR Schur index in the presence of $\mathbb{S}^{\mathbf{n}}$ is evaluated as

$$
\mathcal{I}_{\mathrm{IR}}^{\mathbb{S}^{\mathbf{n}}}(q ; \mathbf{x})=\mathcal{N}_{\mathbf{n}}(q)\left(\mathcal{I}_{\mathrm{vec}}(q)\right)^{\frac{N(N-1)}{2}} \cdot \lim _{\mathbf{y} \rightarrow \mathbf{y}_{\mathbf{n}}^{*}}\left(\mathcal{I}_{\mathrm{UV}}(q ; \mathbf{x}, \mathbf{y}) \prod_{1 \leq i \leq j \leq N-1}\left(1-\prod_{\ell=i}^{j}\left(q^{n_{\ell}} y^{\alpha_{\ell}}\right)\right)\right)
$$

where $\mathcal{N}_{\mathbf{n}}(q)$ is a factor of the form $a q^{b}$ for $a, b \in \mathbb{R}$ so that the $q$-expansion of the index starts with 1. Note here that $\mathbf{y}_{(1,1, \cdots, 1)}^{*}=\mathbf{y}^{*}$, and therefore $\mathbf{n}=(1, \cdots, 1)$ corresponds to the case without surface operators.

In section 3, we study the Schur index with surface operator insertions for the $\left(A_{N-1}, A_{M-1}\right)$ theories with $\operatorname{gcd}(N, M)=1$, via the Higgsing prescription. In section 4, we turn to the $\left(A_{N-1}, A_{N(k-1)-1}\right)$ theories.

\footnotetext{
${ }^{21}$ As shown in section 4.3 of [52], this result can be regarded as a consequence of the quantum DrinfeldSokolov reduction for the associated chiral algebra.
} 


\section{$3\left(A_{N-1}, A_{M-1}\right)$ theories with $\operatorname{gcd}(N, M)=1$}

In this section, we study the Schur indices of the $\left(A_{N-1}, A_{M-1}\right)$ theories with $\operatorname{gcd}(N, M)=1$ in the presence of various surface operators. The case of $N=2$ was carefully studied in [18].

\subsection{Modules in $\mathcal{W}_{N}$ minimal models}

Since the chiral algebra associated with the $\left(A_{N-1}, A_{M-1}\right)$ theory is conjectured in [26] to be the $(N, M+N) \mathcal{W}_{N}$ minimal model, we here review the modules in the $\mathcal{W}_{N}$ minimal models. The $\mathcal{W}_{N}$ Minimal models, dating back to the celebrated work of [53], are a special class of chiral algebras, which have only finite number of non-degenerate modules. Interestingly, the vacuum modules of these chiral algebras correspond to the $\left(A_{N-1}, A_{M-1}\right)$ $\mathrm{AD}$ theories for $\operatorname{gcd}(N, M)=1$ [26]. Since these chiral algebras are relatively simple, they are a good starting point to study the relation between the non-vacuum modules and the surface operators in four dimensions. We here give a brief review of the irreducible modules of the $\mathcal{W}_{N}$ minimal model.

The $\mathcal{W}_{N}$ minimal models are usually labeled by three positive integer parameters, $N$, $P$ and $Q$ (with $P<Q$ and $\operatorname{gcd}(P, Q)=1$ ). $N$ denotes the rank of the global part of the underlying $\mathcal{W}$-algebra. Each module of the model is further labeled by two $N$-dim positive-integer-valued vectors, $\vec{n}=\left(n_{i}\right)_{i=1, \ldots, N}$ and $\vec{n}^{\prime}=\left(n_{i}^{\prime}\right)_{i=1, \ldots, N}$, with the constraints

$$
\sum_{i=1}^{N} n_{i}=Q, \quad \sum_{i=1}^{N} n_{i}^{\prime}=P .
$$

We focus on the special case $P=N$, in which case all $n_{i}^{\prime}$ 's are forced to take the value $n_{i}^{\prime}=1$. Therefore, the modules we are interested in are parameterized by $N$ positive integers summed to be $Q$. Sometimes, it is also convenient to write $Q=N+M$. It is well-known that there is a level-rank duality [54] between a rank- $N$ minimal model labeled by $(P=N, Q=N+M)$ and a rank- $M$ minimal model labeled by $(P=M, Q=N+M)$, as one can see from the expression of minimal model central charge,

$$
c=(N-1)\left(1-\frac{(P-Q)^{2}}{P Q} N(N+1)\right) .
$$

The conformal weight of the module labeled by $\vec{n}$ is given by

$$
h(\vec{n})=\frac{1}{2(N+M)}\left(\sum_{i=1}^{N-1} i(N-i)\left(s_{i}^{2}-M s_{i}\right)+2 \sum_{i<j}^{N-1} i(N-j) s_{i} s_{j}\right),
$$

where we used the notation introduced in the previous section $s_{i}=n_{i}-1$. Note that the above expression is invariant under the permutations among $\tilde{n}_{i}$ 's. Therefore, independent modules are labeled by $\vec{n}$ such that $n_{i} \leq n_{i+1}$ for $i=1, \ldots, N-1$. The character of the module labeled by $\vec{n}$ is given by

$$
\chi_{\vec{n}}^{(N, N+M)}(q)=\frac{\left(q^{N+M} ; q^{N+M}\right)^{N-1} \prod_{i, j}\left(q^{a_{i, j}} ; q^{N+M}\right) \prod_{i, j}\left(q^{N+M-a_{i, j}} ; q^{N+M}\right)}{(q ; q)^{N-1}},
$$


where we used the notation

$$
a_{i, j}=n_{j}+n_{j+1}+\cdots+n_{j+i-1},
$$

for $i, j \in \mathbb{N}$ such that $i+j \leq N$.

\subsection{Review of $\left(A_{1}, A_{2 k}\right)$ series}

Let us first review the $\left(A_{1}, A_{2 k}\right)$ AD theories, whose Schur index with surface operator insertions has been evaluated in [18]. The associated chiral algebra is the Virasoro minimal model labeled by $(P=2, Q=2 k+3)$.

The class $\mathcal{S}$ construction of the $\left(A_{1}, A_{2 k}\right)$ theory involves a Riemann surface $\mathcal{C}_{\text {IR }}$ which is a sphere with one irregular puncture. Therefore the TQFT expression for the Schur index of the $\left(A_{1}, A_{2 k}\right)$ theory is given by

$$
\mathcal{I}_{\left(A_{1}, A_{2 n}\right)}(q)=\sum_{R}\left(C_{R}\right)^{-1} \tilde{f}_{R}^{\left(A_{1}, A_{2 k}\right)}(q),
$$

where $R$ runs over the irreducible representations of $\mathfrak{s u}(2)$, and the expressions for $\left(C_{R}\right)^{-1}$ and $\widetilde{f}_{R}^{\left(A_{1}, A_{2 k}\right)}$ are shown in (2.1) and (2.6). The above expression for the Schur index was shown to be equivalent to the vacuum character of Virasoro algebra for $P=2$ and $Q=2 n+3[18,26,43]$.

The Schur index with surface operator insertions can be evaluated via the Higgsing prescription reviewed in section 2.2. We first consider a UV SCFT associated with the Riemann surface $\mathcal{C}_{\mathrm{UV}}$ which is a sphere with the same irregular puncture and an additional (full) regular puncture. ${ }^{22}$ This UV SCFT is called the $\left(A_{1}, D_{2 k+3}\right)$ theory, whose Schur index is evaluated as $[26,43]$

$$
\mathcal{I}_{\left(A_{1}, D_{2 k+3}\right)}(q ; y)=\sum_{R} \tilde{f}_{R}^{\left(A_{1}, A_{2 k}\right)}(q) f_{R}^{\text {full }}(q ; y),
$$

where $y$ is the fugacity for the flavor $\mathrm{SU}(2)$ symmetry associated with the regular puncture, and the expression for $f_{R}^{\text {full }}(q ; y)$ is shown in (2.3). According to the Higgsing prescription, the Schur index of the IR SCFT in the presence of the surface operator, $\mathbb{S}^{(n)}$, is evaluated as

$$
\mathcal{I}_{\left(A_{1}, A_{2 k}\right)}^{\mathbb{S}(n)}(q)=\mathcal{N}_{n}(q) \mathcal{I}_{\mathrm{vec}}(q) \cdot \lim _{y \rightarrow q^{\frac{n}{2}}}\left(\left(1-q^{n} y^{-2}\right) \mathcal{I}_{\mathrm{UV}}(q ; y)\right)
$$

where $n$ is the label of the surface operator, and $\mathcal{N}(q)$ is a factor of the form $a q^{b}$ for $a, b \in \mathbb{R}$ so that the $q$-expansion of the index starts with 1 . Note that the condition (2.14) now reads $y^{2}=q^{n}$, and therefore is equivalent to $y=q^{\frac{n}{2}}$. The wave function for the full puncture $f_{R}^{\text {full }}(q ; y)=\chi_{R}^{\mathrm{SU}(2)}(y) /(q ; q)\left(q y^{2} ; q\right)\left(q y^{-2} ; q\right)$ has a pole at this point, which is the contribution of the would-be NG multiplet, as reviewed in section 2.2. Since this pole is canceled by $\left(1-q^{n} y^{-2}\right)$ in $(3.8)$, the IR index $\mathcal{I}_{\left(A_{1}, A_{2 k}\right)}^{\mathbb{S}^{(n)}}$ is well-defined and evaluated as [18]

$$
\mathcal{I}_{\left(A_{1}, A_{2 k}\right)}^{\mathbb{S}^{(n)}}(q)=2^{-1}(-1)^{\alpha} q^{\beta} \mathcal{N}_{n}(q) \chi_{(\bar{n}, 2 k+3-\bar{n})}^{(2,2 k+3)}(q)
$$

\footnotetext{
${ }^{22}$ For $N=2$, the only regular puncture is the full puncture.
} 
where $\bar{n}=n \bmod (2 k+3)$, and $\alpha$ and $\beta$ are respectively an integer and a half-integer determined by $n$ and $k$. Since the $q$-series of $\chi_{(\bar{n}, 2 k+3-\bar{n})}^{(2,2 k+3)}(q)$ starts with 1 , the factor $\mathcal{N}_{n}(q)$ is fixed as $\mathcal{N}_{n}(q)=2(-1)^{-\alpha} q^{-\beta}$, which implies

$$
\mathcal{I}_{\left(A_{1}, A_{2 k}\right)}^{\mathbb{S}^{(n)}}(q)=\chi_{(\bar{n}, 2 k+3-\bar{n})}^{(2,2 k+3)}(q) .
$$

This shows that the surface operator $\mathbb{S}^{(n)}$ corresponds to the $(\bar{n}, Q-\bar{n})$ module of the $(2, Q)$ Virasoro minimal model. In particular, $\mathbb{S}^{(n)}$ and $\mathbb{S}^{(n+Q)}$ lead to the same Schur index.

\section{$3.3 \quad\left(A_{N-1}, A_{M-1}\right)$ series with $\operatorname{gcd}(N, M)=1$}

We now consider the generalization of the above discussion to the $\left(A_{N-1}, A_{M-1}\right)$ theories for $N$ and $M$ such that $\operatorname{gcd}(N, M)=1$. We show that the Schur indices with surface operator insertions reproduce all and only the characters of irreducible representations of the $\mathcal{W}_{N}$ minimal model for $(P, Q)=(N, N+M)$.

The $\left(A_{N-1}, A_{M-1}\right)$ theories are of class $\mathcal{S}$ and associated with $\mathcal{C}_{\mathrm{IR}}$ which is a sphere with one irregular puncture. As reviewed in section 2.1, this class $\mathcal{S}$ description implies that the Schur index without surface operators is written as

$$
\mathcal{I}_{\left(A_{N-1}, A_{M-1}\right)}(q)=\sum_{R}\left(C_{R}\right)^{-1} \widetilde{f}_{R}^{\left(A_{N-1}, A_{M-1}\right)}(q),
$$

where $\widetilde{f}\left(A_{N-1}, A_{M-1}\right)$ is given in $(2.5)$ if $\operatorname{gcd}(N, M)=1$. We now consider an UV SCFT associated with $\mathcal{C}_{\mathrm{UV}}$ which is obtained by adding a full regular puncture to $\mathcal{C}_{\mathrm{IR}}$. Its Schur index is given by

$$
\mathcal{I}_{\mathrm{UV}}(q ; \mathbf{y})=\sum_{R} \tilde{f}_{R}^{\left(A_{N-1}, A_{M-1}\right)}(q) f_{R}^{\mathrm{full}}(q, \mathbf{y})
$$

where $f_{R}^{\text {full }}$ is given by (2.2). As shown in section 4.3 of [55], this can be rewritten as ${ }^{23}$

$$
\mathcal{I}_{\mathrm{UV}}(q ; \mathbf{y})=P . E .\left[\left(\frac{q}{1-q}-\frac{q^{M+N}}{1-q^{M+N}}\right) \chi_{\mathrm{adj}}^{\mathrm{SU}(N)}(\mathbf{y})\right] .
$$

Note here that, in terms of the formal exponential $y^{\lambda}$ such that $y_{i}=y^{-\omega_{i}}$ for the fundamental weights $\omega_{i}$ of $\mathrm{SU}(N)$, the character $\chi_{\operatorname{adj}}^{\mathrm{SU}(N)}(\mathbf{y})$ is written as

$$
\chi_{\mathrm{adj}}^{\mathrm{SU}(N)}(\mathbf{y})=N-1+\sum_{1 \leq i \leq j \leq N-1}\left(y^{\alpha_{i}+\alpha_{i+1}+\cdots+\alpha_{j}}+y^{-\left(\alpha_{i}+\alpha_{i+1}+\cdots+\alpha_{j}\right)}\right),
$$

where $\alpha_{i}$ are the simple roots of $\mathrm{SU}(N)$. Therefore the UV index (3.13) is expressed as

$$
\begin{aligned}
\mathcal{I}_{\mathrm{UV}}(q ; \mathbf{y})= & \frac{\left(q^{M+N} ; q^{M+N}\right)^{N-1}}{(q ; q)^{N-1}} \\
& \times \prod_{1 \leq i \leq j \leq N-1} \frac{\left(q^{M+N} y^{\alpha_{i}+\alpha_{i+1}+\cdots+\alpha_{j}} ; q^{M+N}\right)\left(q^{M+N} y^{-\left(\alpha_{i}+\alpha_{i+1}+\cdots+\alpha_{j}\right)} ; q^{M+N}\right)}{\left(q y^{\alpha_{i}+\alpha_{i+1}+\cdots+\alpha_{j}} ; q\right)\left(q y^{-\left(\alpha_{i}+\alpha_{i+1}+\cdots+\alpha_{j}\right)} ; q\right)} .
\end{aligned}
$$

\footnotetext{
${ }^{23}$ To be precise, this is written as P.E. $\left[\frac{q-q^{M+N}}{(1-q)\left(1-q^{M+N}\right)} \chi_{\mathrm{adj}}^{\mathrm{SU}(N)}(\mathbf{y})\right]$ in [55]. This rewriting can be achieved by using the identity $\sum_{R} \chi_{R}^{\mathrm{SU}(N)}(\mathbf{z}) \chi_{R}^{\mathrm{SU}(N)}(\mathbf{y})=\Delta(\mathbf{z})^{-1} \delta(\mathbf{z}-\mathbf{y})$.
} 
Let us now evaluate the Schur index of the $\left(A_{N-1}, A_{M-1}\right)$ theory in the presence of a surface operator $\mathbb{S}^{\mathbf{n}}$. Via the Higgsing prescription, it is evaluated as

$$
\mathcal{I}_{\left(A_{N-1}, A_{M-1}\right)}^{\mathbb{S n}^{\mathbf{n}}}(q)=\left(\mathcal{I}_{\mathrm{vec}}(q)\right)^{\frac{N(N-1)}{2}} \lim _{\mathbf{y} \rightarrow \mathbf{y}_{\mathbf{n}}^{*}}\left(\mathcal{I}_{\mathrm{UV}}(q ; \mathbf{y}) \prod_{1 \leq i \leq j \leq N-1}\left(1-\prod_{k=i}^{j}\left(q^{n_{k}} y^{\alpha_{k}}\right)\right)\right),
$$

where $\mathbf{y}_{\mathbf{n}}^{*}$ is the simultaneous solution to the equations (2.14). Recall that the simple roots are related to the fundamental weights by $\alpha_{i}=\sum_{j=1}^{N-1} c_{i j} \omega_{j}$, where $\left(c_{i j}\right)$ is the Cartan matrix. Therefore, the equations (2.14) are equivalent to

$$
y^{\alpha_{\ell}}=q^{-n_{\ell}} \quad \text { for } \quad \ell=1, \cdots, N-1 .
$$

We see that the factor $\prod_{1 \leq i \leq j \leq N-1}\left(q y^{\alpha_{i}+\cdots+\alpha_{j}} ; q\right)$ in the denominator of (3.15) contains a divergent factor canceled by $\prod_{1 \leq i \leq j \leq N-1}\left(1-\prod_{k=1}^{j}\left(q y^{\alpha_{k}}\right)\right)$ in (3.16). In particular,

$$
\lim _{\mathbf{y} \rightarrow \mathbf{y}_{\mathbf{n}}^{*}} \frac{\prod_{1 \leq i \leq j \leq N-1}\left(1-\prod_{k=1}^{j}\left(q y^{\alpha_{k}}\right)\right)}{\prod_{1 \leq i \leq j \leq N-1}\left(q y^{\left(\alpha_{i}+\cdots+\alpha_{j}\right)} ; q\right)}=\prod_{1 \leq i \leq j \leq N-1} \frac{(-1)^{n_{i}+\cdots+n_{j}-1} q^{\frac{\left(n_{i}+\cdots+n_{j}\right)\left(n_{i}+\cdots+n_{j}-1\right)}{2}}}{(q ; q)_{n_{i}+\cdots+n_{j}-1}(q ; q)},
$$

where $(x ; q)_{k} \equiv \prod_{i=1}^{k}\left(1-q^{i-1} x\right)$. Therefore, the IR index is evaluated as

$$
\begin{aligned}
\mathcal{I}_{\left(A_{N-1}, A_{M-1}\right)}^{\mathbb{S}^{\mathrm{n}}}(q)=\left(\mathcal{I}_{\mathrm{vec}}(q)\right)^{\frac{N(N-1)}{2}} & \frac{\left(q^{M+N} ; q^{M+N}\right)^{N-1}}{(q ; q)^{N-1}} \\
\times \prod_{1 \leq i \leq j \leq N-1}\{ & \left\{(-1)^{n_{i}+\cdots+n_{j}-1} q^{\frac{\left(n_{i}+\cdots+n_{j}\right)\left(n_{i}+\cdots+n_{j}-1\right)}{2}}\right. \\
& \left.\times \frac{\left(q^{M+N+\left(n_{i}+\cdots+n_{j}\right)} ; q^{M+N}\right)\left(q^{M+N-\left(n_{i}+\cdots+n_{j}\right)} ; q^{M+N}\right)}{(q ; q)_{n_{i}+\cdots+n_{j}-1}\left(q^{1+n_{i}+n_{i+1}+\cdots+n_{j}} ; q\right)(q ; q)}\right\} .
\end{aligned}
$$

Using $\mathcal{I}_{\text {vec }}(q)=(q ; q)^{2}$ and the identities

$$
(q ; q)_{n-1}\left(q^{n+1} ; q\right)=\frac{(q ; q)}{\left(1-q^{n}\right)}, \quad\left(1-q^{n}\right)\left(q^{M+N+n} ; q^{M+N}\right)=\left(q^{n} ; q^{M+N}\right),
$$

we can rewrite this as

$$
\begin{aligned}
\mathcal{I}_{\left(A_{N-1}, A_{M-1}\right)}^{\mathbb{S n}^{\mathrm{n}}}(q)= & \frac{\left(q^{M+N} ; q^{M+N}\right)^{N-1}}{(q ; q)^{N-1}} \prod_{1 \leq i \leq j \leq N-1}\left(q^{n_{i}+\cdots+n_{j}} ; q^{M+N}\right)\left(q^{M+N-\left(n_{i}+\cdots+n_{j}\right)} ; q^{M+N}\right) \\
& \times \mathcal{N}_{\mathbf{n}}(q) \prod_{1 \leq i \leq j \leq N-1}(-1)^{n_{i}+\cdots+n_{j}-1} q^{\frac{\left(n_{i}+\cdots+n_{j}\right)\left(n_{i}+\cdots+n_{j}-1\right)}{2}} .
\end{aligned}
$$

Recall that $\mathcal{N}_{\mathbf{n}}(q)$ is a factor which makes the $q$-expansion of the IR index start with 1 . In our case, it is fixed as

$$
\mathcal{N}_{\mathbf{n}}(q) \equiv \prod_{1 \leq i \leq j \leq N-1}(-1)^{n_{i}+\cdots+n_{j}-1} q^{-\frac{\left(n_{i}+\cdots+n_{j}\right)\left(n_{i}+\cdots+n_{j}-1\right)}{2}} .
$$


Then we see that the IR index $\mathcal{I}_{\left(A_{N-1}, A_{M-1}\right)}^{\mathbb{S}^{\mathrm{n}}}(q)$ is precisely equivalent to the character of the irreducible $\mathcal{W}_{N}$ module (3.4) for $\vec{n} \equiv\left(\mathbf{n}, N+M-\sum_{i=1}^{N-1} n_{i}\right)$. Note here that, up to the prefactor absorbed in $\mathcal{N}_{\mathbf{n}}(q)$, the character (3.4) is invariant under $n_{i} \rightarrow n_{i}+N+M$ for ${ }^{\forall} i$. Therefore, the two surface operators, $\mathbb{S}^{\mathbf{n}}$ and $\mathbb{S}^{\mathbf{n}+(N+M) \mathbf{1}}$, are related to the same module of the $\mathcal{W}_{N}$ minimal model. For $N=2$ this degeneracy was already noted in [18], and we here naturally generalize it to the $\mathcal{W}_{N}$ minimal model.

\section{$4 \quad\left(A_{N-1}, A_{N(k-1)-1}\right)$ theories}

In this section, we study the Schur indices of the $\left(A_{N-1}, A_{N(k-1)-1}\right)$ theories in the presence of surface operators. The chiral algebras associated with these theories were conjectured to be the logarithmic $\mathcal{B}(k)_{A_{N-1}}$ algebras [31]. The Schur indices with surface operator insertions for $N=2$ and $k=2,3$ and 4 were already computed and shown to be identical to the characters of modules of the associated chiral algebra in [18]. We here generalize it to the whole $\left(A_{N}, A_{N(k-1)-1}\right)$ theories, and particularly show for the $\left(A_{1}, A_{2 k-3}\right)$ theories that the indices are identical to the characters of modules of the $\mathcal{B}(k)_{A_{1}}$ algebra.

\subsection{Logarithmic $\mathcal{B}(k)_{A_{N-1}}$ algebra}

The chiral algebra associated with the $\left(A_{N-1}, A_{N(k-1)-1}\right)$ theory was conjectured in [31] to be the logarithmic $\mathcal{B}(k)_{A_{N-1}}$ algebra constructed in the paper. The $\mathcal{B}(k)_{A_{N-1}}$ is a nice generalization of the $\mathcal{B}_{k}$ algebra constructed in [39], the latter of which is conjectured to be isomorphic to the $W_{k-1}^{(2)}$ algebra [56]. ${ }^{24}$ In this sub-section we briefly review the structure of the $\mathcal{B}(k)_{A_{N-1}}$ algebra, following [31].

The $\mathcal{B}(k)_{A_{N-1}}$ is a vertex operator algebra defined as the kernel of screening operators in a lattice vertex operator algebra. ${ }^{25}$ Let $\mathcal{F}_{\lambda}$ be the weight- $\lambda$ Fock module of the Heisenberg vertex operator algebra of rank $(N-1)$, and $P$ be the weight lattice of $\mathrm{SU}(N)$. We then define $\mathcal{M}(k, \mu) \equiv \bigcap_{j=1}^{N-1} \operatorname{ker}_{\mathcal{F}_{\sqrt{k} \mu}} e_{0}^{-\alpha_{j} / \sqrt{k}}$ for $k \in \mathbb{N}$ and $\mu \in P$, where $e_{0}^{-\alpha_{j} / \sqrt{k}}$ is the screening operator associated with the $j$-th simple root of $\mathrm{SU}(N)$. The $\mathcal{B}(k)_{A_{N-1}}$ algebra is decomposed as

$$
\mathcal{B}(k)_{A_{N-1}} \simeq \bigoplus_{\mu \in P} \mathcal{M}(k, \mu) \otimes \mathcal{F}_{\sqrt{-k} \mu}
$$

The character of $\mathcal{M}(k, \mu)$ is evaluated as

$$
\chi(\mathcal{M}(k, \mu))=\frac{q^{\frac{\rho^{2}}{2 k}}}{\eta(q)^{N-1}} \sum_{\lambda \in P^{+} \cap(Q+\mu)}\left(q^{\frac{k}{2}(\lambda+\rho)^{2}} m_{\lambda}(\mu) \sum_{w \in W} \epsilon(w) q^{-\langle\rho, w(\lambda+\rho)\rangle}\right),
$$

where $\eta(q) \equiv q^{\frac{1}{24}} \prod_{i=1}^{\infty}\left(1-q^{i}\right), P^{+}$denotes the set of dominant weights, $Q$ is the root lattice, and $m_{\lambda}(\mu)$ counts the multiplicity of weight $\mu$ in the highest weight representation of $\operatorname{SU}(N)$

\footnotetext{
${ }^{24}$ The author of [31] indeed constructed the $\mathcal{B}(k)_{\mathcal{Q}}$ algebra for a general simply-laced Lie algebra $\mathcal{Q}$. We focus on the case $\mathcal{Q}=A_{N-1}$ in this paper.

${ }^{25}$ The author of [31] also provided a construction of $\mathcal{B}(k)_{\mathcal{Q}}$ in terms of the "corner VOA" studied in [57, 58].
} 
associated with $\lambda$. On the other hand, the character of the Fock module is given by

$$
\chi\left(\mathcal{F}_{\sqrt{-k \mu} \mu}\right)=\frac{q^{-\frac{k \mu^{2}}{2}} x^{\mu}}{\eta(q)^{N-1}} .
$$

The tensor product decomposition (4.1) then implies that the vacuum character of the $\mathcal{B}(k)_{A_{N-1}}$ is given by

$$
\chi\left(\mathcal{B}(k)_{A_{N-1}}\right)=\sum_{\mu \in P} \chi(\mathcal{M}(k, \mu)) \chi\left(\mathcal{F}_{\sqrt{-k} \mu}\right),
$$

which was proven in [31] to coincide with the Schur index of the $\left(A_{N-1}, A_{N(k-1)-1}\right)$ theory, up to the normalization. In the case of $N=2$, a series of $\mathcal{B}(k)_{A_{1}}$ modules, $W_{s}$, is constructed in $[29,39]$. These modules are of the form

$$
W_{s}=\bigoplus_{r \in \mathbb{Z}} M_{r+1, s} \otimes \mathcal{F}_{\sqrt{-k} r}
$$

in terms of $M_{r, s} \equiv \bigoplus_{\ell=0}^{\infty} L\left(h_{r+2 \ell, s}, c_{k}\right)$ for $r \geq 1$ and $M_{r, s} \equiv \bigoplus_{\ell=0}^{\infty} L\left(h_{r-1-2 \ell, k-s}, c_{k}\right)$ for $r \leq 0$, where $L\left(h_{r, s}, c_{k}\right)$ is the simple highest weight module of Virasoro algebra at the highest weight $h_{r, s} \equiv\left((k r-s)^{2}-(k-1)^{2}\right) / 4 k$ and the Virasoro central charge $c_{k} \equiv 1-6(k-1)^{2} / k$. The character of $M_{r+1, s}$ is evaluated in the reference as

$$
\chi\left(M_{r+1, s}\right)=\left\{\begin{array}{ll}
\frac{1}{\eta(q)} \sum_{\ell \geq 0}\left(q^{k\left(\frac{r+1}{2}+\ell-\frac{s}{2 k}\right)^{2}}-q^{k\left(\frac{r+1}{2}+\ell+\frac{s}{2 k}\right)^{2}}\right) & \text { for } \quad r \geq 0 \\
\frac{1}{\eta(q)} \sum_{\ell \leq 0}\left(q^{k\left(\frac{r-1}{2}+\ell+\frac{s}{2 k}\right)^{2}}-q^{k\left(\frac{r+1}{2}+\ell-\frac{s}{2 k}\right)^{2}}\right) & \text { for } \quad r \leq-1
\end{array} .\right.
$$

\subsection{Schur indices with surface operator insertions}

We now study the Schur indices of the $\left(A_{N-1}, A_{N(k-1)-1}\right)$ theory with surface operator insertions. To that end, we first consider the UV SCFT associated to a sphere $\mathcal{C}_{\text {UV }}$ with one irregular puncture and a full regular puncture. We then Higgs the $\mathrm{SU}(N)$ flavor symmetry associated with the full puncture to recover the $\left(A_{N-1}, A_{N(k-1)-1}\right)$ theory as the IR SCFT. The Schur index of the UV SCFT is evaluated via the TQFT description as

$$
\mathcal{I}_{\mathrm{UV}}(q ; \mathbf{x} ; \mathbf{y})=\sum_{R} \widetilde{f}_{R}^{\left(A_{N-1}, A_{N(k-1)-1}\right)}(q ; \mathbf{x}) f_{R}^{\text {full }}(q ; \mathbf{y})
$$

where $R$ runs over the irreducible representations of $\mathfrak{s u}(N)$, and $\mathbf{y}=\left(y_{1}, \cdots, y_{N-1}\right)$ is the fugacity for the flavor $\mathrm{SU}(N)$ symmetry arising from the full puncture.

According to the Higgsing prescription, the IR index in the presence of a surface operator $\mathbb{S}^{\mathbf{n}}$ is evaluated as $(2.15)$, where $\mathbf{y}=\mathbf{y}_{\mathbf{n}}^{*}$ is again the solution to (2.14). To evaluate this limit, we first note that

$$
\begin{aligned}
& \left(\mathcal{I}_{\mathrm{vec}}(q)\right)^{\frac{N(N-1)}{2}} \lim _{\mathbf{y} \rightarrow \mathbf{y}_{\mathbf{n}}^{*}}\left(f_{R}^{\mathrm{full}}(q ; \mathbf{y}) \prod_{1 \leq i \leq j \leq N-1}\left(1-\prod_{k=i}^{j}\left(q^{n_{k}} y^{\alpha_{k}}\right)\right)\right) \\
& =\left(\prod_{1 \leq i \leq j \leq N-1}(-1)^{n_{i}+\cdots+n_{j}-1} q^{\frac{\left(n_{i}+\cdots+n_{j}\right)\left(n_{i}+\cdots+n_{j}-1\right)}{2}}\right) \frac{q^{\left(\nu_{\mathbf{n}}, \rho\right)}}{(q ; q)^{N-1}} \delta\left(q^{-\nu_{\mathbf{n}}}\right) \chi_{R}^{\mathrm{SU}(N)}\left(q^{-\nu_{\mathbf{n}}}\right),
\end{aligned}
$$


where we use the short-hand notations

$$
\nu_{\mathbf{n}} \equiv \sum_{i=1}^{N-1} n_{i} \omega_{i}, \quad \delta(x) \equiv x^{\rho} \prod_{\beta \in \Delta_{+}}\left(1-x^{-\beta}\right),
$$

and recall that in our notation $\left(q^{\nu}\right)^{\beta}=q^{(\nu, \beta)}$ and therefore $\chi_{R}^{\mathrm{SU}(N)}\left(q^{\nu}\right)=\sum_{\mu \in \lambda} m_{\lambda}(\mu) q^{(\nu, \mu)}$ with $\lambda$ being the highest weight of $R$. The identity (4.8) directly follows from the expression (2.2) for the wave function associated with the full puncture. Substituting it and (4.7) into the equation (2.15), we find that the IR index in the presence of the surface operator $\mathbb{S}^{\mathbf{n}}$ is given by

$$
\mathcal{I}_{\left(\mathrm{A}_{\mathrm{N}-1}, \mathrm{~A}_{\mathrm{N}(\mathbf{k}-1)-1} \mathbb{S}^{\mathbf{n}}\right.}(q ; \mathbf{x})=\frac{1}{(q ; q)^{N-1}} \sum_{R} \widetilde{f}_{R}^{\left(A_{N-1}, A_{N(k-1)-1}\right)}(q ; \mathbf{x}) q^{\left(\nu_{\mathbf{n}}, \rho\right)} \delta\left(q^{-\nu_{\mathbf{n}}}\right) \chi_{R}^{\mathrm{SU}(N)}\left(q^{-\nu_{\mathbf{n}}}\right),
$$

where we used the fact that $\mathcal{N}_{\mathbf{n}}(q)$ in $(2.15)$ is again fixed as in (3.22). To relate this to the character of a module of the associated chiral algebra $\mathcal{B}(k)_{A_{N-1}}$, we use the formulae

$$
\begin{aligned}
\widetilde{f}_{R}^{\left(A_{N-1}, A_{N(k-1)-1}\right)}(q ; \mathbf{x}) & =\frac{1}{(q ; q)^{N-1}} q^{\frac{k}{2}(\lambda, \lambda+2 \rho)} \sum_{\mu \in Q+\lambda} m_{\lambda}(\mu) q^{-\frac{k}{2} \mu^{2}} x^{\mu} \\
\delta\left(q^{-\nu_{\mathbf{n}}}\right) \chi_{R}^{\mathrm{SU}(N)}\left(q^{-\nu_{\mathbf{n}}}\right) & =\sum_{w \in W} \epsilon(w) q^{-\left(\nu_{\mathbf{n}}, w(\lambda+\rho)\right)}
\end{aligned}
$$

where $\lambda$ is the highest weight of $R, W$ is the Weyl group of $\mathfrak{s u}(N)$, and $\epsilon(w)$ is the sign of the Weyl reflection. The first rewriting (4.11) was obtained in [31] while the second one (4.12) follows from the Weyl's character formula. Substituting these two into (4.10) and performing a small computation as in section 3 of [31], we obtain

$$
\begin{aligned}
& \mathcal{I}_{\left(\mathrm{A}_{\mathrm{N}-1}, \mathrm{~A}_{\mathrm{N}(\mathrm{k}-1)-1}\right)}^{\mathbb{S}^{\mathbf{n}}}(q ; \mathbf{x}) \\
& =\frac{q^{-\frac{k}{2} \rho^{2}-\left(\nu_{\mathbf{n}}, \rho\right)}}{(q ; q)^{2(N-1)}} \sum_{\mu \in P}\left(\sum_{\lambda \in P^{+} \cap(Q+\mu)} q^{\frac{k}{2}(\lambda+\rho)^{2}} m_{\lambda}(\mu) q^{-\frac{k}{2} \mu^{2}} x^{\mu} \sum_{w \in W} \epsilon(w) q^{-\left(\nu_{\mathbf{n}}, w(\lambda+\rho)\right)}\right) .
\end{aligned}
$$

In terms of (4.3) and

$$
\chi(k, \mu, \nu) \equiv \frac{q^{\frac{\nu^{2}}{2 k}}}{\eta(q)^{N-1}} \sum_{\lambda \in P^{+} \cap(Q+\mu)} q^{\frac{k}{2}(\lambda+\rho)^{2}} m_{\lambda}(\mu) \sum_{w \in W} \epsilon(w) q^{-(\nu, w(\lambda+\rho))},
$$

we finally have

$$
\mathcal{I}_{\left(\mathrm{A}_{\mathrm{N}-1}, \mathrm{~A}_{\mathrm{N}(\mathrm{k}-1)-1}\right)}^{\mathbb{S}^{\mathbf{n}}}(q ; \mathbf{x})=q^{-\frac{\rho^{2}+\nu_{\mathbf{n}}^{2}+2 k\left(\nu_{\mathbf{n}}, \rho\right)}{2 k}-\frac{N-1}{12}} \sum_{\mu \in P} \chi\left(k, \mu, \nu_{\mathbf{n}}\right) \chi\left(\mathcal{F}_{\sqrt{-k} \mu}\right) .
$$




\subsection{Comparison to the characters of $\mathcal{B}(k)_{A_{N-1}}$ modules}

Let us now compare the above result with the character of modules of the associated chiral algebra $\mathcal{B}(k)_{A_{N-1}}$. We start with the case of $N=2$, and write $\mu=r \omega_{1}$ and $\nu=s \omega_{1}$ in terms of the fundamental weight $\omega_{1}$ of $\mathrm{SU}(2)$. In this case, the expression for $\chi(k, \mu, \nu)$ reduces to

$$
\chi(k, \mu, \nu)=\frac{q^{\frac{s^{2}}{4 k}}}{\eta(q)} \sum_{\ell=0}^{\infty} q^{\frac{k}{4}(2 \ell+|r|+1)^{2}}\left(q^{-\frac{s(2 \ell+|r|+1)}{2}}-q^{\frac{s(2 \ell+|r|+1)}{2}}\right) .
$$

After a small computation, we see that this is precisely equivalent to the character of the Virasoro module $M_{r+1, s}$ shown in (4.6), i.e. ${ }^{26}$

$$
\chi(k, \mu, \nu)=\chi\left(M_{r+1, s}\right) .
$$

This and (4.15) imply that

$$
\mathcal{I}_{\left(\mathrm{A}_{1}, \mathrm{~A}_{2 \mathrm{k}-3}\right)}^{\mathbb{S}^{(n)}}(q ; x)=q^{-\frac{1+\nu^{2}+2 k \nu}{4 k}-\frac{1}{12}} \sum_{r=-\infty}^{\infty} \chi\left(M_{r+1, n}\right) \chi\left(\mathcal{F}_{\sqrt{-k} r}\right)=q^{-\frac{1+\nu^{2}+2 k \nu}{4 k}-\frac{1}{12}} \chi\left(W_{n}\right),
$$

where $W_{n}$ is the $\mathcal{B}(k)_{A_{1}}$-module $W_{s}$ for $s=n$ reviewed in (4.5). Hence, up to the prefactor $q^{-\frac{1+\nu^{2}+2 k \nu}{4 k}-\frac{1}{12}}$, the IR index $\mathcal{I}_{\left(\mathrm{A}_{1}, \mathrm{~A}_{2 \mathrm{k}-3}\right)}^{\mathbb{S}^{(n)}}(q ; \mathbf{x})$ in the presence of the surface operator $\mathbb{S}^{(n)}$ is identical to the character of the $\mathcal{B}(k)_{A_{1}}$ module $W_{n}$. In particular, the label $n$ of the surface operator is now interpreted as the label of the $\mathcal{B}(k)_{A_{1}}$-module. The different prefactor arises here since we normalize our IR index so that its $q$-expansion starts with 1 . This correspondence between the surface operators $\mathbb{S}^{(n)}$ and the $\mathcal{B}(k)_{A_{1}}$-modules $W_{n}$ can be regarded as a generalization of the detailed discussions on $k=2,3$ and 4 in [18].

For general $N>2$, modules of the $\mathcal{B}(k)_{A_{N-1}}$ are not well-studied. However, after the above success in the $N=2$ case, it is natural to expect that there exists a higher-rank generalization, $\mathcal{M}(k, \mu, \nu)$, of the module $M_{r, s}$ so that its character $\chi(\mathcal{M}(k, \mu, \nu))$ coincides with (4.14). Note that $M_{r, s}$ can be realized as the kernel of a screening charge $\mathcal{Q}_{-}^{[s]}[38]$, where $\mathcal{Q}_{-}^{[s]}$ is the zero mode of the product of $s$ screening currents acting on the Fock module $\mathcal{F}_{\alpha_{r, s}}$ with $\alpha_{r, s} \equiv \frac{1-r}{2} \sqrt{2 k}-\frac{1-s}{2} \sqrt{\frac{2}{k}}$. Therefore we expect that $\mathcal{M}(k, \mu, \nu)$ is also realized in the same way as the kernel of a screening charge. We then conjecture that there exists a module $W_{\nu}$ of $\mathcal{B}(k)_{A_{N-1}}$ which is decomposed as

$$
W_{\nu}=\bigoplus_{\mu \in P} \mathcal{M}(k, \mu, \nu) \otimes \mathcal{F}_{\sqrt{-k} \mu} .
$$

This means that $\mathcal{I}_{\left(\mathrm{A}_{\mathrm{N}-1}, \mathrm{~A}_{\mathrm{N}(\mathrm{k}-1)-1}\right)}^{\mathbb{S n}^{\mathrm{n}}}(q ; \mathbf{x})$ coincides with the character of $W_{\nu_{\mathbf{n}}}$ up to the prefactor $q^{-\frac{\rho^{2}+\nu_{\mathbf{n}}^{2}+2 k\left(\nu_{\mathbf{n}}, \rho\right)}{2 k}-\frac{N-1}{12}}$. We will leave a careful study of this conjecture for future work.

\footnotetext{
${ }^{26}$ Note here that, since it is a module of the Virasoro algebra at $c=1-6(k-1)^{2} / k$, the $M_{\mu+1, \nu}$ implicitly depends on $k$.
} 


\section{Conclusion and discussion}

In this article, we have studied the Schur indices of two series of $\left(A_{N-1}, A_{M-1}\right)$ ArgyresDouglas theories in the presence of surface operators; one for $N$ and $M$ satisfying $\operatorname{gcd}(N, M)=1$ and the other for $M=N(k-1)$. We have used the Higgsing prescription proposed in [42] and shown, for all theories in the first series and theories with $N=2$ in the second series, that the Schur indices in the presence of the surface operators $\mathbb{S}^{\mathbf{n}}$ are identical to the characters of non-vacuum modules of the associated chiral algebras in the sense of [15], which explicitly confirms the recent proposal on the general correspondence between surface operators and modules of chiral algebras [18]. We have also given a conjecture on modules of the $\mathcal{B}(k)_{A_{N-1}}$ algebra based on our results on the second series of the Argyres-Douglas theories for $N>2$.

As mentioned in section 4.3, non-degenerate modules $M_{r, s}$ in Virasoro minimal models can be expressed in terms of the kernel and image of screening charges $\mathcal{Q}_{-}^{[s]}$ (see [38]). We expect a more general corresponding principle exists between poles and screening charges in the VOA approach. We leave it as a future work together with the extension to other theories such as the Argyres-Douglas theories of type $\left(A_{k-1}, D_{n-1}\right)$.

The Macdonald limit of the superconformal index [4] with surface operator inserted is also an interesting object to study, as it gives a refined character of the space of the same set of local operators. As discussed in [15, 30, 59, 60], the refinement is realized by a new grading for the number of "basic" operators used to produce a descendant state, which in Virasoro minimal models counts the number of $L_{-}{ }_{n}$ 's in each state. At least for the vacuum module, a clear way to add this new grading in the computation of the character can be found in the POSET approach to minimal models [61, 62], whose generalization to higer-rank cases and non-vacuum modules is worth studying.

Last but not least, a similar Higgsing procedure also exists in the computation of the instanton partition function (or the $S^{4}$ partition function) [63], as it can be uplift to the superconformal index of $5 \mathrm{~d} \mathcal{N}=1$ theories. In terms of the topological string, this Higgsing procedure corresponds to the geometric transition and surface operators obtained this way are the so-called monodromy defects studied in [64, 65] (see also [66] for an approach from $6 \mathrm{~d} \mathcal{N}=(2,0)$ theories) labeled by a nilpotent elements in $\mathfrak{g}$. When $\mathfrak{g}=\mathfrak{s u}(n)$, nilpotent elements are classified by the partition of $n,\left\{n_{i}\right\}$ with $\sum_{i} n_{i}=n$. On the other hand, modules in minimal models with level-rank duality are also labeled by such a partition. The relation between them, together with its generalization to the other chiral algebras, is an interesting problem to work on.

\section{Acknowledgments}

We would like to thank Tomoyuki Arakawa, Matthew Buican, Thomas Creutzig, Yutaka Matsuo, Jaewon Song and Akihiro Tsuchiya for helpful discussion. T.N. particularly thanks Matthew Buican for various illuminating discussions in many collaborations on the superconformal indices and chiral algebras of Argyres-Douglas theories. The work of T.N. is partially supported by JSPS KAKENHI Grant Number 18K13547. R.Z. is supported by JSPS fellowship for young students. 


\section{A Explicit $q$-series for lower-rank examples}

We here give the explicit $q$-series expression for the Schur indices with surface operator insertions for lower-rank $\left(A_{N-1}, A_{M-1}\right)$ theories with $\operatorname{gcd}(M, N)=1$. In this appendix, we use the fact that the Higgsing prescription gives rise to the following replacement of the wave function for the full regular puncture:

$$
(q ; q)^{|\Delta|} f_{R}^{\text {full }}\left(q, y_{i}\right) \rightarrow f_{\vec{t}}(q) \frac{\chi_{R}\left(q^{t_{i}}\right)}{\chi_{R}\left(q^{\rho}\right)} C_{R}^{-1}, \quad y_{i} \rightarrow q^{t_{i}},
$$

where $|\Delta|=\frac{N(N-1)}{2}$ is the number of roots of $\mathrm{SU}(N)$, and $t_{i}$ are determined by $\mathbf{n}$ so that

$$
n_{i}=\sum_{j} c_{i j} t_{j}
$$

where $\left(c_{i j}\right)$ is the Cartan matrix of $\mathrm{SU}(N)$. This replacement allows us to interpret the factor $f_{\vec{t}}(q) \frac{\chi_{R}\left(q^{t_{i}}\right)}{\chi_{R}\left(q^{\rho}\right)}$ as the contribution from the surface defect $\mathbb{S}^{\mathbf{n}}$ to the Schur index. Especially $f_{\vec{t}}(q)$ does not depend on the representation of $\mathrm{SU}(N)$, and when we take $\left\{q_{i}^{t}\right\}=q^{\rho}$, $\mathbb{S}^{\mathbf{n}}$ becomes a trivial defect with $f_{\vec{t}}(q)=1$.

We need the explicit expression of $\tilde{f}_{R}^{\left(A_{N-1}, A_{M-1}\right)}(q)$ given in (2.5) to perform the $q$ series computation. The wavefunction series $\tilde{f}_{R}^{\left(A_{N-1}, A_{M-1}\right)}(q)$ can be generated from a formal wavefunction $\psi_{R}^{I_{N,-N+1}}(q)$ by

$$
\tilde{f}_{R}^{\left(A_{N-1}, A_{M-1}\right)}(q)=\psi_{R}^{I_{N,-N+1}}\left(q^{N+M}\right),
$$

and as shown in [43], $\psi_{R}^{I_{N,-N+1}}(q)$ can be evaluated to

$$
\psi_{R}^{I_{N,-N+1}}(q)=\sum_{n_{\alpha} \in \mathbb{Z}} \frac{(-1)^{\sum_{\alpha} n_{\alpha}} q^{\sum_{\alpha} \frac{1}{2} n_{\alpha}\left(n_{\alpha}+1\right)}}{|\mathcal{W}|(q ; q)^{\frac{1}{2}(N-1)(N-2)}} \sum_{w \in \mathcal{W}} \epsilon(w) \delta_{w \cdot \lambda(R)=\sum_{\alpha \in \Delta^{+}} n_{\alpha} \alpha}
$$

where $w \cdot \lambda=w(\lambda+\rho)-\rho$ is a shifted Weyl reflection, and $\lambda(R)$ stands for the highest weight of the representation $R$ of $\mathrm{SU}(N)$. With the help of the (generalized) Euler identities given in appendix B, one can explicitly calculate $\psi_{R}^{I_{N,-N+1}}(q)$ for low-dimensional representations $R .{ }^{27}$ In particular, for $N=3$, the closed form of $\psi_{R}^{I_{3,-2}}(q)$ is conjectured in [43] to be

$$
\psi_{\left(\lambda_{1}, \lambda_{2}\right)}^{I_{3,-2}}(q)=\left\{\begin{array}{cc}
q^{k(k+1)+l(l+1)+k l} & \lambda_{1}=3 k, \lambda_{2}=3 l \\
-q^{k^{2}+l^{2}-1+(k-1)(l-1)} & \lambda_{1}=3 k-2, \quad \lambda_{2}=3 l-2, \\
0 & \text { otherwise }
\end{array}\right.
$$

where $\left(\lambda_{1}, \lambda_{2}\right)$ stands for the highest weight of an irreducible representation of $\mathrm{SU}(3)$. Similarly, one can see that in the case of $N=4$, the first several non-trivial contributions, $\psi_{R}^{I_{4},-3}(q)$, come from the trivial representation $\mathbf{1}$, the 15-dim representation $(1,0,1)$,

\footnotetext{
${ }^{27}$ Note that the fundamental weights are expressed in terms of simple roots as $\mu_{1}=\frac{2}{3} \alpha_{1}+\frac{1}{3} \alpha_{2}$, $\mu_{2}=\frac{1}{3} \alpha_{1}+\frac{2}{3} \alpha_{2}$.
} 
two 45-dim representations $(2,1,0)$ and $(0,1,2)$, two 35-dim representations $(4,0,0)$ and $(0,0,4)$ given by $^{28}$

$$
\psi_{(0,0,0)}^{I_{4,-3}}=1, \quad \psi_{(1,0,1)}^{I_{4,-3}}=-q, \quad \psi_{(2,1,0)}^{I_{4,-3}}=\psi_{(0,1,2)}^{I_{4,-3}}=q^{2}, \quad \psi_{(4,0,0)}^{I_{4,-3}}=\psi_{(0,0,4)}^{I_{4,-3}}=-q^{3}, \quad \ldots
$$

We note that the contribution from the $\mathbf{2 0}$-dim representation $(0,2,0)$ also vanishes.

Based on the conjectured Euler identity for $N=5$ given in appendix B, we also list the first several non-trivial wavefunctions for $\psi_{R}^{I_{5,-4}}(q)$ :

$$
\psi_{(0,0,0,0)}^{I_{5,-4}}=1, \quad \psi_{(1,0,0,1)}^{I_{5,-4}}=-q, \quad \psi_{(2,0,1,0)}^{I_{5,-4}}=\psi_{(0,1,0,2)}^{I_{5,-4}}=q^{2}, \quad \ldots
$$

\section{A.1 $\left(A_{2}, A_{n-1}\right)$ series with $\operatorname{gcd}(3, n)=1$}

Let us first examine $\left(A_{2}, A_{n-1}\right)$ theories with $\operatorname{gcd}(3, n)=1$. We start with $\left(A_{2}, A_{3}\right)$ theory, whose Schur index is given by

$$
\begin{aligned}
\mathcal{I}_{\left(A_{2}, A_{3}\right)} & =\frac{1}{\left(q^{2} ; q\right)\left(q^{3} ; q\right)} \sum_{\left(\lambda_{1}, \lambda_{2}\right)} \chi_{\left(\lambda_{1}, \lambda_{2}\right)}\left(q^{\rho}\right) \psi_{\left(\lambda_{1}, \lambda_{2}\right)}^{I_{3,-2}}\left(q^{7}\right) \\
& =\frac{1}{\left(q^{2} ; q\right)\left(q^{3} ; q\right)}\left(1-q^{7} \sum_{\left(w_{1}, w_{2}\right) \in R_{(1,1)}} q^{w_{1}+w_{2}}+\mathcal{O}\left(q^{11}\right)\right) \\
& =\frac{1}{\left(q^{2} ; q\right)\left(q^{3} ; q\right)}\left(1-q^{5}-2 q^{6}-2 q^{7}-2 q^{8}+\mathcal{O}\left(q^{9}\right)\right) \\
& =1+q^{2}+2 q^{3}+3 q^{4}+3 q^{5}+6 q^{6}+7 q^{7}+11 q^{8}+\mathcal{O}\left(q^{9}\right) .
\end{aligned}
$$

It agrees with the vacuum character of the $(P=3, Q=7)$ minimal model.

Recall that the wave function of the regular puncture for $G=\mathrm{SU}(3)$ is given by

$$
f_{R}^{\text {full }}\left(q, y_{1}, y_{2}\right)=\frac{\chi_{R}\left(y_{1}, y_{2}\right)}{(q ; q)^{2}\left(q y_{1}^{2} y_{2}^{-1} ; q\right)\left(q y_{1}^{-1} y_{2}^{2} ; q\right)\left(q y_{1} y_{2} ; q\right)\left(q y_{1}^{-2} y_{2}^{1} ; q\right)\left(q y_{1}^{1} y_{2}^{-2} ; q\right)\left(q y_{1}^{-1} y_{2}^{-1} ; q\right)} .
$$

The insertion of a trivial surface operator corresponds to the reduction ${ }^{29}$

$$
(q ; q)^{6} f_{R}^{\text {full }}\left(q, y_{1}, y_{2}\right) \rightarrow C_{R}^{-1}, \quad y_{1}, y_{2} \rightarrow q,
$$

and thus the Schur index with a trivial surface operator $\mathbb{S}^{\mathbf{n}=(1,1)}$ inserted reproduces the vacuum character.

The first non-trivial pole, that gives rise to a non-vacuum character, appears at $y_{1}=q^{\frac{4}{3}}$ and $y_{2}=q^{\frac{5}{3}}$. Sitting on this pole, the regular puncture reduces to the factor,

$$
(q ; q)^{6} f_{R}^{\text {full }}\left(q, y_{1}, y_{2}\right) \rightarrow \frac{1+q+q^{2}}{\left(q^{2} ; q\right)\left(q^{3} ; q\right)} \chi_{R}\left(q^{\frac{4}{3}}, q^{\frac{5}{3}}\right),
$$

\footnotetext{
${ }^{28} \mathrm{In} \mathrm{SU}(4)$, the fundamental weights are given by $\mu_{1}=\frac{3}{4} \alpha_{1}+\frac{1}{2} \alpha_{2}+\frac{1}{4} \alpha_{3}, \mu_{2}=\frac{1}{2} \alpha_{1}+\alpha_{2}+\frac{1}{2} \alpha_{3}$, $\mu_{3}=\frac{1}{4} \alpha_{1}+\frac{1}{2} \alpha_{2}+\frac{3}{4} \alpha_{3}$.

${ }^{29}$ As explained in section 3.3 , we need to compensate a factor $\mathcal{N}_{\mathbf{n}}(q)$ in this calculation so that the IR index starts from 1. For our convenience, we include this factor implicitly in this kind of reduction denoted by $\rightarrow$.
} 
and the corresponding Schur index of $\left(A_{2}, A_{3}\right)$ with this insertion can be computed as

$$
\begin{aligned}
\mathcal{I}_{\left(A_{2}, A_{3}\right)}^{\mathbb{S}^{(1,2)}}(q) & =\frac{1+q+q^{2}}{\left(q^{2} ; q\right)\left(q^{3} ; q\right)}\left(1-q^{7} \sum_{\left(w_{1}, w_{2}\right) \in R_{(1,1)}} q^{\frac{4}{3} w_{1}+\frac{5}{3} w_{2}}+q^{14} \sum_{\substack{\left(w_{1}, w_{2}\right) \in R_{(3,0)} \\
\left(w_{1}, w_{2}\right) \in R_{(0,3)}}} q^{\frac{4}{3} w_{1}+\frac{5}{3} w_{2}}+\mathcal{O}\left(q^{20}\right)\right) \\
& =\frac{1+q+q^{2}}{\left(q^{2} ; q\right)\left(q^{3} ; q\right)}\left(1-q^{4}-q^{5}-q^{6}-2 q^{7}-q^{8}+\mathcal{O}\left(q^{10}\right)\right) \\
& =1+q+2 q^{2}+3 q^{3}+5 q^{4}+7 q^{5}+11 q^{6}+14 q^{7}+21 q^{8}+28 q^{9}+\mathcal{O}\left(q^{10}\right) .
\end{aligned}
$$

It agrees with the character of the $(1,2,4)$ module in the $(P=3, Q=7)$ minimal model. Similarly, the pole at $y_{1}=q^{\frac{5}{3}}$ and $y_{2}=q^{\frac{7}{3}}$ leads to

$$
(q ; q)^{6} f_{R}^{\text {full }}\left(q, y_{1}, y_{2}\right) \rightarrow \frac{\left(1+q^{2}\right)\left(1+q+q^{2}\right)}{\left(q^{2} ; q\right)\left(q^{3} ; q\right)} \chi_{R}\left(q^{\frac{5}{3}}, q^{\frac{7}{3}}\right), \quad y_{1} \rightarrow q^{\frac{5}{3}}, y_{2} \rightarrow q^{\frac{7}{3}}
$$

and

$$
\begin{aligned}
\mathcal{I}_{\left(A_{2}, A_{3}\right)}^{\mathbb{S}(1,3)}(q) & =\frac{\left(1+q^{2}\right)\left(1+q+q^{2}\right)}{\left(q^{2} ; q\right)\left(q^{3} ; q\right)}\left(1-q^{7} \sum_{\left(w_{1}, w_{2}\right) \in R_{(1,1)}} q^{\frac{5}{3} w_{1}+\frac{7}{3} w_{2}}+q^{14} \sum_{\substack{\left(w_{1}, w_{2}\right) \in R_{(3,0)} \\
\left(w_{1}, w_{2}\right) \in R_{(0,3)}}} q^{\frac{5}{3} w_{1}+\frac{7}{3} w_{2}}+\mathcal{O}\left(q^{17}\right)\right) \\
& =\frac{\left(1+q^{2}\right)\left(1+q+q^{2}\right)}{\left(q^{2} ; q\right)\left(q^{3} ; q\right)}\left(1-q^{3}-q^{4}-q^{6}-q^{7}-q^{8}+q^{9}+\mathcal{O}\left(q^{10}\right)\right) \\
& =1+q+3 q^{2}+3 q^{3}+6 q^{4}+8 q^{5}+13 q^{6}+17 q^{7}+25 q^{8}+33 q^{9}+\mathcal{O}\left(q^{10}\right) .
\end{aligned}
$$

It reproduces the character of the $(1,3,3)$ module in the $(P=3, Q=7)$ minimal model. The pole at $x_{1}=q^{\frac{7}{3}}$ and $x_{2}=q^{\frac{8}{3}}$ leads to the factor

$$
f_{\left(\frac{7}{3}, \frac{8}{3}\right)}(q)=\left(1+q+q^{2}\right)\left(1+q+q^{2}+q^{3}+q^{4}\right)
$$

and the index

$$
\begin{aligned}
& \mathcal{I}_{\left(A_{2}, A_{3}\right)}^{\mathbb{S}^{(2,3)}}(q)=\frac{\left(1+q+q^{2}\right)\left(1+q+q^{2}+q^{3}+q^{4}\right)}{\left(q^{2} ; q\right)\left(q^{3} ; q\right)} \\
& \times\left(1-q^{7} \sum_{\left(w_{1}, w_{2}\right) \in R_{(1,1)}} q^{\frac{7}{3} w_{1}+\frac{8}{3} w_{2}}+q^{14} \sum_{\substack{\left(w_{1}, w_{2}\right) \in R_{(3,0)} \\
\left(w_{1}, w_{2}\right) \in R_{(0,3)}}} q^{\frac{7}{3} w_{1}+\frac{8}{3} w_{2}}+\mathcal{O}\left(q^{15}\right)\right) \\
& =\frac{\left(1+q+q^{2}\right)\left(1+q+q^{2}+q^{3}+q^{4}\right)}{\left(q^{2} ; q\right)\left(q^{3} ; q\right)}\left(1-q^{2}-q^{4}-q^{5}+q^{6}-q^{7}+q^{9}+\mathcal{O}\left(q^{10}\right)\right) \\
& =1+2 q+3 q^{2}+5 q^{3}+8 q^{4}+11 q^{5}+17 q^{6}+24 q^{7}+34 q^{8}+47 q^{9}+\mathcal{O}\left(q^{10}\right),
\end{aligned}
$$


which agrees with the character of the $(2,2,3)$ module in the $(P=3, Q=7)$ minimal model. In summary, we obtain the following correspondence between poles and modules,

$$
\begin{array}{lll}
y_{1}=q, \quad y_{2}=q & \leftrightarrow & (1,1,5) \text { vacuum module, } \\
y_{1}=q^{\frac{4}{3}}, y_{2}=q^{\frac{5}{3}} & \leftrightarrow & (1,2,4) \text { module, } \\
y_{1}=q^{\frac{5}{3}}, y_{2}=q^{\frac{7}{3}} & \leftrightarrow & (1,3,3) \text { module, } \\
y_{1}=q^{\frac{7}{3}}, y_{2}=q^{\frac{8}{3}} & \leftrightarrow & (2,2,3) \text { module },
\end{array}
$$

which exactly reproduces the correspondence rule (2.14) obtained from the general discussion.

One can confirm that other poles do not give rise to any new characters, for example we have

$$
\mathcal{I}_{\left(A_{2}, A_{3}\right)}^{\mathbb{S}^{(2,4)}}(q)=\mathcal{I}_{\left(A_{2}, A_{3}\right)}^{\mathbb{S}^{(1,2)}}(q), \quad \mathcal{I}_{\left(A_{2}, A_{3}\right)}^{\mathbb{S}^{(2,2)}}(q)=\mathcal{I}_{\left(A_{2}, A_{3}\right)}^{\mathbb{S}^{(2,3)}}(q), \quad \mathcal{I}_{\left(A_{2}, A_{3}\right)}^{\mathbb{S}^{(1,4)}}(q)=\mathcal{I}_{\left(A_{2}, A_{3}\right)}^{\mathbb{S}^{(1,2)}}(q), \quad \ldots
$$

The above fact reflects the permutation symmetry among $n_{1} \leftrightarrow n_{2} \leftrightarrow n_{3}$ in $W_{3}$ minimal models.

In the same way, we can check that the correspondence between poles and modules in $(P=3, Q=8)$ minimal model (the dual of $\left(A_{2}, A_{4}\right)$ theory) is given by ${ }^{30}$

$$
\begin{array}{lll}
y_{1}=q, y_{2}=q & \leftrightarrow & (1,1,6) \text { vacuum module } \\
y_{1}=q^{\frac{5}{3}}, y_{2}=q^{\frac{7}{3}} & \leftrightarrow & (1,3,4) \text { module } \\
y_{1}=q^{\frac{7}{3}}, y_{2}=q^{\frac{8}{3}} & \leftrightarrow & (2,3,3) \text { module } \\
y_{1}=q^{\frac{8}{3}}, y_{2}=q^{\frac{10}{3}} & \leftrightarrow & (2,2,4) \text { module. }
\end{array}
$$

In the $(P=3, Q=10)$ minimal model, the correspondence is given by

$$
\begin{aligned}
y_{1}=q, \quad y_{2}=q & \leftrightarrow \quad(1,1,8) \text { vacuum module, } \\
y_{1}=q^{\frac{4}{3}}, y_{2}=q^{\frac{5}{3}} & \leftrightarrow \quad(1,2,7) \text { module, } \\
y_{1}=q^{\frac{5}{3}}, y_{2}=q^{\frac{7}{3}} & \leftrightarrow \quad(1,3,6) \text { module, } \\
y_{1}=q^{\frac{7}{3}}, y_{2}=q^{\frac{8}{3}} & \leftrightarrow \quad(2,3,5) \text { module, } \\
y_{1}=q^{\frac{8}{3}}, y_{2}=q^{\frac{10}{3}} & \leftrightarrow \quad(2,4,4) \text { module, } \\
y_{1}=q^{\frac{10}{3}}, y_{2}=q^{\frac{11}{3}} & \leftrightarrow \quad(3,3,4) \text { module, } \\
y_{1}=q^{\frac{10}{3}}, y_{2}=q^{\frac{14}{3}} & \leftrightarrow \quad(2,2,6) \text { module, } \\
y_{1}=q^{\frac{13}{3}}, y_{2}=q^{\frac{14}{3}} & \leftrightarrow \quad(1,4,5) \text { module. }
\end{aligned}
$$

The remaining poles such as $y_{1}=q^{\frac{11}{3}}, y_{2}=q^{\frac{13}{3}}$ give rise to the character, for example, of $(2,3,5)$ module again.

\footnotetext{
${ }^{30}$ We checked this correspondence up to $\mathcal{O}\left(q^{11}\right)$ order. We will always check this kind of correspondence up to this order throughout the paper, unless claimed otherwise.
} 
It is clear from the above explicit computations that the pole position $y_{1}=q^{t_{1}}$ and $y_{2}=q^{t_{2}}$ in the $\left(A_{2}, A_{n-1}\right)$ series indeed corresponds to the $\left(n_{1}, n_{2}, n_{3}\right)$ module in the dual $W_{3}$ minimal model following the rule (2.14), i.e.

$$
n_{1}=\log _{q}\left(y_{1}^{2} y_{2}^{-1}\right)=2 t_{1}-t_{2}, \quad n_{2}=\log _{q}\left(y_{2}^{2} y_{1}^{-1}\right)=2 t_{2}-t_{1}
$$

and $n_{3}$ is determined from $n_{1}+n_{2}+n_{3}=Q$, together with a permutation symmetry among $\left(n_{1}, n_{2}, n_{3}\right)$.

We can also check the level-rank duality between $\left(A_{1}, A_{2}\right)$ theory and $\left(A_{2}, A_{1}\right)$ theory for surface operators. The Schur index of $\left(A_{2}, A_{1}\right)$ computed in the TQFT way,

$$
\begin{aligned}
\mathcal{I}_{\left(A_{2}, A_{1}\right)} & =\frac{1}{\left(q^{2} ; q\right)\left(q^{3} ; q\right)} \sum_{\left(\lambda_{1}, \lambda_{2}\right)} \chi_{\left(\lambda_{1}, \lambda_{2}\right)}\left(q^{\rho}\right) f_{\left(\lambda_{1}, \lambda_{2}\right)}^{I_{3,-2}}\left(q^{5}\right) \\
& =\frac{1}{\left(q^{2} ; q\right)\left(q^{3} ; q\right)}\left(1-q^{5} \sum_{\left(w_{1}, w_{2}\right) \in R_{(1,1)}} q^{w_{1}+w_{2}}+q^{10} \sum_{\left(w_{1}, w_{2}\right) \in R_{(3,0)}, R_{(0,3)}} q^{w_{1}+w_{2}}+\mathcal{O}\left(q^{15}\right)\right) \\
& =\frac{1}{\left(q^{2} ; q\right)\left(q^{3} ; q\right)}\left(1-q^{3}-2 q^{4}-2 q^{5}-2 q^{6}-q^{7}+2 q^{7}+2 q^{8}+\mathcal{O}\left(q^{9}\right)\right) \\
& =1+q^{2}+q^{3}+q^{4}+2 q^{5}+2 q^{6}+2 q^{7}+3 q^{8}+\mathcal{O}\left(q^{9}\right),
\end{aligned}
$$

matches with the $\left(A_{1}, A_{2}\right)$ index. The pole at $y_{1}=q^{\frac{4}{3}}, y_{2}=q^{\frac{5}{3}}$ reproduces the character of the $(2,3)$ module of the $(P=2, Q=5)$ Lee-Yang model, or equivalently the $(1,2,2)$ module of the level-rank dual model $(P=3, Q=5)$.

$$
\begin{aligned}
\mathcal{I}_{\left(A_{2}, A_{1}\right)}^{\mathbb{S}^{(1,2)}}(q) & =\frac{1+q+q^{2}}{\left(q^{2} ; q\right)\left(q^{3} ; q\right)}\left(1-q^{2}-q^{3}-q^{4}-q^{5}+q^{7}+q^{8}+3 q^{9}+\mathcal{O}\left(q^{10}\right)\right) \\
& =1+q+q^{2}+q^{3}+2 q^{4}+2 q^{5}+3 q^{6}+3 q^{7}+4 q^{8}+5 q^{9}+\mathcal{O}\left(q^{10}\right) \\
& =\mathcal{I}_{\left(A_{1}, A_{2}\right)}^{\mathbb{S}^{(2)}}(q):
\end{aligned}
$$

Similarly, the index at the pole $y_{1}=q^{\frac{5}{3}}, y_{2}=q^{\frac{7}{3}}$,

$$
\begin{aligned}
\mathcal{I}_{\left(A_{2}, A_{1}\right)}^{\mathbb{S}(1,3)}(q) & =\frac{\left(1+q^{2}\right)\left(1+q+q^{2}\right)}{\left(q^{2} ; q\right)\left(q^{3} ; q\right)}\left(1-q-q^{2}+q^{3}-q^{4}-q^{5}+q^{6}+2 q^{7}+q^{10}+\mathcal{O}\left(q^{11}\right)\right) \\
& =1+q^{2}+q^{3}+q^{4}+q^{5}+2 q^{6}+2 q^{7}+3 q^{8}+3 q^{9}+4 q^{10}+\mathcal{O}\left(q^{11}\right) \\
& =\mathcal{I}_{\left(A_{1}, A_{2}\right)}(q)
\end{aligned}
$$

corresponds to the character of the $(1,3,1)$ module, i.e. the vacuum $(1,1,3)$ module of the $(P=3, Q=5)$ minimal model.

\section{A.2 $\left(A_{3}, A_{4}\right)$ theory and $\left(A_{4}, A_{5}\right)$ theory}

We now consider a rank-three example, the $\left(A_{3}, A_{4}\right)$ theory, and a rank-four example, the $\left(A_{4}, A_{5}\right)$ theory. 
First the Schur index of the $\left(A_{3}, A_{4}\right)$ theory matches with the character of the $(1,1,1,6)$ vacuum module of $(P=4, Q=9)$ minimal model:

$$
\begin{aligned}
\mathcal{I}_{\left(A_{3}, A_{4}\right)}(q) & =\frac{1}{\left(q^{2} ; q\right)\left(q^{3} ; q\right)\left(q^{4} ; q\right)} \sum_{\left(\lambda_{1}, \lambda_{2}, \lambda_{3}\right)} \chi_{\left(\lambda_{1}, \lambda_{2}, \lambda_{3}\right)}\left(q^{\rho}\right) f_{\left(\lambda_{1}, \lambda_{2}, \lambda_{3}\right)}^{I_{4,-3}}\left(q^{9}\right) \\
& =\frac{1}{\left(q^{2} ; q\right)\left(q^{3} ; q\right)\left(q^{4} ; q\right)}\left(1-q^{6}-2 q^{7}-3 q^{8}-3 q^{9}-3 q^{10}+\mathcal{O}\left(q^{11}\right)\right) \\
& =1+q^{2}+2 q^{3}+4 q^{4}+5 q^{5}+9 q^{6}+12 q^{7}+21 q^{8}+29 q^{9}+44 q^{10}+\mathcal{O}\left(q^{11}\right) .
\end{aligned}
$$

It also corresponds to the Higgsed Schur index with an additional regular puncture with respect to the pole $y_{1}=q^{\frac{3}{2}}, y_{2}=q^{2}$ and $y_{3}=q^{\frac{3}{2}}$, i.e. $y=q^{\rho}$. This pole is translated according to the dictionary $(2.14)$ to the trivial surface operator $\mathbb{S}^{(1,1,1)}$.

The pole at $\left(y_{1}, y_{2}, y_{3}\right)=\left(q^{\frac{7}{4}}, q^{\frac{5}{2}}, q^{\frac{9}{4}}\right)$ gives rise to the character of the $(1,1,2,5)$ module of $(P=4, Q=9) W_{4}$ minimal model,

$$
\begin{aligned}
\mathcal{I}_{\left(A_{3}, A_{4}\right)}^{\mathbb{S}^{(1,1,2)}}(q) & =\frac{1+q+q^{2}+q^{3}}{\left(q^{2} ; q\right)\left(q^{3} ; q\right)\left(q^{4} ; q\right)} \sum_{\left(\lambda_{1}, \lambda_{2}, \lambda_{3}\right)} \chi_{\left(\lambda_{1}, \lambda_{2}, \lambda_{3}\right)}\left(q^{\frac{7}{4}}, q^{\frac{5}{2}}, q^{\frac{9}{4}}\right) f_{\left(\lambda_{1}, \lambda_{2}, \lambda_{3}\right)}^{I_{4,-3}}\left(q^{9}\right) \\
& =\frac{1+q+q^{2}+q^{3}}{\left(q^{2} ; q\right)\left(q^{3} ; q\right)\left(q^{4} ; q\right)}\left(1-q^{5}-q^{6}-2 q^{7}-2 q^{8}-3 q^{9}-2 q^{10}+\mathcal{O}\left(q^{11}\right)\right) \\
& =1+q+2 q^{2}+4 q^{3}+7 q^{4}+11 q^{5}+19 q^{6}+28 q^{7}+44 q^{8}+65 q^{9}+97 q^{10}+\mathcal{O}\left(q^{11}\right),
\end{aligned}
$$

as

$$
f_{\left(\frac{7}{4}, \frac{5}{2}, \frac{9}{4}\right)}(q)=1+q+q^{2}+q^{3} .
$$

We also checked the following correspondence in the $\left(A_{3}, A_{4}\right)$ theory,

$$
\begin{array}{llll}
\left(y_{1}, y_{2}, y_{3}\right) & =\left(q^{2}, q^{3}, q^{3}\right) & \leftrightarrow & (1,1,3,4) \text { module }, \\
\left(y_{1}, y_{2}, y_{3}\right)=\left(q^{\frac{9}{4}}, q^{\frac{7}{2}}, q^{\frac{15}{4}}\right) & \leftrightarrow & (1,1,4,3) \text { module, } \\
\left(y_{1}, y_{2}, y_{3}\right)=\left(q^{\frac{9}{4}}, q^{\frac{7}{2}}, q^{\frac{11}{4}}\right) & \leftrightarrow & (1,2,2,4) \text { module, } \\
\left(y_{1}, y_{2}, y_{3}\right)=\left(q^{\frac{5}{2}}, q^{4}, q^{\frac{7}{2}}\right) & \leftrightarrow & (1,2,3,3) \text { module, } \\
\left(y_{1}, y_{2}, y_{3}\right)=\left(q^{3}, q^{4}, q^{3}\right) & \leftrightarrow & (2,2,2,3) \text { module. }
\end{array}
$$

It exactly matches with the translation rule (2.14) and that modules in the minimal model share the same label $\mathbf{n}=\left(n_{1}, n_{2}, n_{3}\right)$ with the surface operator $\mathbb{S}^{\mathbf{n}}$.

In the $\left(A_{4}, A_{5}\right)$ theory, by using the expressions of the factor $f_{\vec{t}}(q)$, e.g.

$$
\begin{aligned}
& f_{\left(\frac{11}{5}, \frac{17}{5}, \frac{18}{5}, \frac{14}{5}\right)}(q)=1+q+q^{2}+q^{3}+q^{4}, \\
& f_{\left(\frac{12}{5}, \frac{19}{5}, \frac{21}{5}, \frac{18}{5}\right)}(q)=\left(1+q+q^{2}+q^{3}+q^{4}\right) \frac{1-q^{6}}{1-q^{2}},
\end{aligned}
$$

we checked the correspondence

$$
\begin{array}{llll}
\left(y_{1}, y_{2}, y_{3}, y_{4}\right)=\left(q^{2}, q^{3}, q^{3}, q^{2}\right) & \leftrightarrow & (1,1,1,1,7) \text { vacuum module, } \\
\left(y_{1}, y_{2}, y_{3}, y_{4}\right)=\left(q^{\frac{11}{5}}, q^{\frac{17}{5}}, q^{\frac{18}{5}}, q^{\frac{14}{5}}\right) & \leftrightarrow & (1,1,1,2,6) \text { module } \\
\left(y_{1}, y_{2}, y_{3}, y_{4}\right)=\left(q^{\frac{12}{5}}, q^{\frac{19}{5}}, q^{\frac{21}{5}}, q^{\frac{18}{5}}\right) & \leftrightarrow & (1,1,1,3,5) \text { module. }
\end{array}
$$




\section{B Generalized Euler identities}

In fact the Euler identity,

$$
\sum_{n \in \mathbb{Z}}(-1)^{n} q^{\frac{3}{2} n^{2} \pm \frac{1}{2} n}=(q ; q)
$$

is a special case of the Jacobi triple identity,

$$
\sum_{n \in \mathbb{Z}} p^{n^{2}} z^{n}=\prod_{n>0}\left(1-p^{2 n}\right)\left(1+p^{2 n-1} z\right)\left(1+p^{2 n-1} z^{-1}\right),
$$

with $p=q^{\frac{3}{2}}$ and $z=-q^{ \pm \frac{1}{2}}$.

For the Generalized Euler identity used in $G=\mathrm{SU}(4)$, we can rewrite it as

$$
\begin{aligned}
& \sum_{n_{1}, n_{2}, n_{3}}(-1)^{n_{1}+n_{2}} q^{\frac{3}{2} n_{1}^{2}+\frac{3}{2} n_{2}^{2}+2 n_{3}^{2}+2 n_{1} n_{3}+2 n_{2} n_{3}+n_{1} n_{2}-\frac{1}{2} n_{1}-\frac{1}{2} n_{2}-n_{3}} \\
& =\sum_{\substack{n_{1}, n_{2}, n_{3} \\
=}}(-1)^{n_{1}+n_{2}} q^{\left(n_{1}+n_{3}\right)^{2}+\left(n_{2}+n_{3}\right)^{2}+\frac{1}{2}\left(n_{1}+n_{2}\right)^{2}-\frac{1}{2}\left(n_{1}+n_{2}+2 n_{3}\right)} \\
& =\sum_{\substack{k_{1}, k_{2}, k_{3} \in \mathbb{Z} \\
k_{1}+k_{2}+k_{3} \in 2 \mathbb{Z}}}(-1)^{k_{3}} q^{k_{1}^{2}+k_{2}^{2}+\frac{1}{2} k_{3}^{2}-\frac{1}{2}\left(k_{1}+k_{2}\right)} \\
& =\sum_{\substack{k_{1}, k_{2}, k_{3} \in \mathbb{Z} \\
k_{1}+k_{2}+k_{3} \in 2 \mathbb{Z}}}(-1)^{k_{1}+k_{2}} q^{k_{1}^{2}+k_{2}^{2}+\frac{1}{2} k_{3}^{2}-\frac{1}{2}\left(k_{1}+k_{2}\right)},
\end{aligned}
$$

where we set $k_{1}=n_{1}+n_{3}, k_{2}=n_{2}+n_{3}$ and $k_{3}=n_{1}+n_{2}$. Using the equality in the last line, we further have

$$
\sum_{\substack{k_{1}, k_{2}, k_{3} \in \mathbb{Z} \\ k_{1}+k_{2}+k_{3} \in 2 \mathbb{Z}}}(-1)^{k_{3}} q^{k_{1}^{2}+k_{2}^{2}+\frac{1}{2} k_{3}^{2}-\frac{1}{2}\left(k_{1}+k_{2}\right)}=\sum_{k_{1}, k_{2}, k_{3} \in \mathbb{Z}} \frac{1+(-1)^{k_{1}+k_{2}+k_{3}}}{2}(-1)^{k_{3}} q^{k_{1}^{2}+k_{2}^{2}+\frac{1}{2} k_{3}^{2}-\frac{1}{2}\left(k_{1}+k_{2}\right)} .
$$

Substituting $(p, z)=\left(q, \pm q^{-\frac{1}{2}}\right),\left(q^{\frac{1}{2}}, \mp 1\right)$ into the Jacobi triple identity, we obtain

$$
\begin{aligned}
\sum_{k \in \mathbb{Z}} q^{k^{2}-\frac{1}{2} k} & =\prod_{n>0}\left(1-q^{2 n}\right)\left(1+q^{n-\frac{1}{2}}\right), \\
\sum_{k \in \mathbb{Z}}(-1)^{k} q^{\frac{1}{2} k^{2}} & =\prod_{n>0}\left(1-q^{n}\right)\left(1-q^{n-\frac{1}{2}}\right)^{2}, \\
\sum_{k \in \mathbb{Z}}(-1)^{k} q^{k^{2}-\frac{1}{2} k} & =\prod_{n>0}\left(1-q^{2 n}\right)\left(1-q^{n-\frac{1}{2}}\right), \\
\sum_{k \in \mathbb{Z}} q^{\frac{1}{2} k^{2}} & =\prod_{n>0}\left(1-q^{n}\right)\left(1+q^{n-\frac{1}{2}}\right)^{2},
\end{aligned}
$$

and therefore

$$
\begin{aligned}
& \sum_{n_{1}, n_{2}, n_{3}}(-1)^{n_{1}+n_{2}} q^{\frac{3}{2} n_{1}^{2}+\frac{3}{2} n_{2}^{2}+2 n_{3}^{2}+2 n_{1} n_{3}+2 n_{2} n_{3}-n_{1} n_{2}-\frac{1}{2} n_{1}-\frac{1}{2} n_{2}-n_{3}} \\
& =\sum_{n_{1}, n_{2}, n_{3}}(-1)^{n_{3}} q^{\frac{3}{2} n_{1}^{2}+\frac{3}{2} n_{2}^{2}+2 n_{3}^{2}+2 n_{1} n_{3}+2 n_{2} n_{3}-n_{1} n_{2}-\frac{1}{2} n_{1}-\frac{1}{2} n_{2}-n_{3}} \\
& =\prod_{n>0}\left(1-q^{n}\right)\left(1-q^{2 n}\right)^{2}\left(1-q^{2 n-1}\right)^{2}=(q ; q)^{3} .
\end{aligned}
$$


For $G=\mathrm{SU}(5)$, we conjecture that the following generalized Euler identity holds,

$$
\begin{aligned}
(q ; q)^{6}=\sum_{n_{1,2,3,4,5,6} \in \mathbb{Z}} & (-1)^{n_{1}+n_{2}+n_{3}+n_{6}} q^{\frac{3}{2} n_{1}^{2}+\frac{3}{2} n_{2}^{2}+\frac{3}{2} n_{3}^{2}+2 n_{4}^{2}+2 n_{5}^{2}+\frac{5}{2} n_{6}^{2}} \\
& \times q^{n_{1} n_{2}+2 n_{1} n_{4}+n_{1} n 5+2 n_{1} n_{6}+n_{2} n_{3}+2 n_{2} n_{4}+2 n_{2} n_{5}+2 n_{2} n_{6}} \\
& \times q^{n_{3} n_{4}+2 n_{3} n_{5}+2 n_{3} n_{6}+2 n_{4} n_{5}+3 n_{4} n_{6}+3 n_{5} n_{6}} \\
& \times q^{-\frac{1}{2} n_{1}-\frac{1}{2} n_{2}-\frac{1}{2} n_{3}-n_{4}-n_{5}-\frac{3}{2} n_{6}} .
\end{aligned}
$$

Open Access. This article is distributed under the terms of the Creative Commons Attribution License (CC-BY 4.0), which permits any use, distribution and reproduction in any medium, provided the original author(s) and source are credited.

\section{References}

[1] L.F. Alday, D. Gaiotto and Y. Tachikawa, Liouville Correlation Functions from Four-dimensional Gauge Theories, Lett. Math. Phys. 91 (2010) 167 [arXiv:0906.3219] [INSPIRE].

[2] N. Wyllard, $A(N-1)$ conformal Toda field theory correlation functions from conformal $\mathcal{N}=2 \mathrm{SU}(N)$ quiver gauge theories, JHEP 11 (2009) 002 [arXiv:0907.2189] [INSPIRE].

[3] A. Gadde, L. Rastelli, S.S. Razamat and W. Yan, The 4d Superconformal Index from q-deformed 2d Yang-Mills, Phys. Rev. Lett. 106 (2011) 241602 [arXiv:1104.3850] [INSPIRE].

[4] A. Gadde, L. Rastelli, S.S. Razamat and W. Yan, Gauge Theories and Macdonald Polynomials, Commun. Math. Phys. 319 (2013) 147 [arXiv:1110.3740] [inSPIRE].

[5] D. Gaiotto, $N=2$ dualities, JHEP 08 (2012) 034 [arXiv:0904.2715] [INSPIRE].

[6] D. Gaiotto, G.W. Moore and A. Neitzke, Wall-crossing, Hitchin Systems and the WKB Approximation, arXiv:0907.3987 [INSPIRE].

[7] C. Beem, L. Rastelli and B.C. van Rees, More $\mathcal{N}=4$ superconformal bootstrap, Phys. Rev. D 96 (2017) 046014 [arXiv: 1612.02363] [INSPIRE].

[8] A. Gadde, E. Pomoni, L. Rastelli and S.S. Razamat, S-duality and 2d Topological QFT, JHEP 03 (2010) 032 [arXiv:0910.2225] [InSPIRE].

[9] M. Buican, S. Giacomelli, T. Nishinaka and C. Papageorgakis, Argyres-Douglas Theories and S-duality, JHEP 02 (2015) 185 [arXiv:1411.6026] [INSPIRE].

[10] D. Xie and S.-T. Yau, New $\mathcal{N}=2$ dualities, arXiv:1602.03529 [INSPIRE].

[11] D. Xie and S.-T. Yau, Argyres-Douglas matter and $\mathcal{N}=2$ dualities, arXiv:1701.01123 [INSPIRE].

[12] M. Buican, Z. Laczko and T. Nishinaka, $\mathcal{N}=2$ S-duality revisited, JHEP 09 (2017) 087 [arXiv:1706.03797] [INSPIRE].

[13] D. Xie and K. Ye, Argyres-Douglas matter and S-duality: Part II, JHEP 03 (2018) 186 [arXiv:1711.06684] [INSPIRE].

[14] M. Buican and T. Nishinaka, Conformal Manifolds in Four Dimensions and Chiral Algebras, J. Phys. A 49 (2016) 465401 [arXiv: 1603.00887] [INSPIRE]. 
[15] C. Beem, M. Lemos, P. Liendo, W. Peelaers, L. Rastelli and B.C. van Rees, Infinite Chiral Symmetry in Four Dimensions, Commun. Math. Phys. 336 (2015) 1359 [arXiv:1312.5344] [INSPIRE].

[16] M. Buican and Z. Laczko, Nonunitary Lagrangians and unitary non-Lagrangian conformal field theories, Phys. Rev. Lett. 120 (2018) 081601 [arXiv:1711.09949] [INSPIRE].

[17] C. Cordova, D. Gaiotto and S.-H. Shao, Surface Defect Indices and 2d-4d BPS States, JHEP 12 (2017) 078 [arXiv: 1703. 02525] [INSPIRE].

[18] C. Cordova, D. Gaiotto and S.-H. Shao, Surface Defects and Chiral Algebras, JHEP 05 (2017) 140 [arXiv: 1704.01955] [INSPIRE].

[19] C. Cordova, D. Gaiotto and S.-H. Shao, Infrared Computations of Defect Schur Indices, JHEP 11 (2016) 106 [arXiv: 1606. 08429] [INSPIRE].

[20] P.C. Argyres and M.R. Douglas, New phenomena in SU(3) supersymmetric gauge theory, Nucl. Phys. B 448 (1995) 93 [hep-th/9505062] [INSPIRE].

[21] P.C. Argyres, M.R. Plesser, N. Seiberg and E. Witten, New $\mathcal{N}=2$ superconformal field theories in four-dimensions, Nucl. Phys. B 461 (1996) 71 [hep-th/9511154] [INSPIRE].

[22] T. Eguchi, K. Hori, K. Ito and S.-K. Yang, Study of $\mathcal{N}=2$ superconformal field theories in four-dimensions, Nucl. Phys. B 471 (1996) 430 [hep-th/9603002] [INSPIRE].

[23] S. Cecotti, A. Neitzke and C. Vafa, R-Twisting and $4 d / 2 d$ Correspondences, arXiv: 1006.3435 [INSPIRE].

[24] G. Bonelli, K. Maruyoshi and A. Tanzini, Wild Quiver Gauge Theories, JHEP 02 (2012) 031 [arXiv:1112.1691] [INSPIRE].

[25] D. Xie, General Argyres-Douglas Theory, JHEP 01 (2013) 100 [arXiv:1204.2270] [InSPIRE].

[26] C. Cordova and S.-H. Shao, Schur Indices, BPS Particles and Argyres-Douglas Theories, JHEP 01 (2016) 040 [arXiv: 1506.00265] [INSPIRE].

[27] K. Ito and H. Shu, ODE/IM correspondence and the Argyres-Douglas theory, JHEP 08 (2017) 071 [arXiv: 1707.03596] [INSPIRE].

[28] K. Ito, M. Mariño and H. Shu, TBA equations and resurgent Quantum Mechanics, JHEP 01 (2019) 228 [arXiv: 1811.04812] [INSPIRE].

[29] T. Creutzig, W-algebras for Argyres-Douglas theories, arXiv:1701.05926 [INSPIRE].

[30] C. Beem and L. Rastelli, Vertex operator algebras, Higgs branches and modular differential equations, JHEP 08 (2018) 114 [arXiv:1707.07679] [INSPIRE].

[31] T. Creutzig, Logarithmic W-algebras and Argyres-Douglas theories at higher rank, JHEP 11 (2018) 188 [arXiv: 1809.01725] [INSPIRE].

[32] J. Choi and T. Nishinaka, On the chiral algebra of Argyres-Douglas theories and S-duality, JHEP 04 (2018) 004 [arXiv:1711.07941] [INSPIRE].

[33] M. Buican and T. Nishinaka, On the superconformal index of Argyres-Douglas theories, $J$. Phys. A 49 (2016) 015401 [arXiv: 1505.05884] [InSPIRE].

[34] M. Buican and T. Nishinaka, On Irregular Singularity Wave Functions and Superconformal Indices, JHEP 09 (2017) 066 [arXiv:1705.07173] [INSPIRE]. 
[35] B.L. Feigin, A.M. Gainutdinov, A.M. Semikhatov and I.Y. Tipunin, Kazhdan-Lusztig correspondence for the representation category of the triplet W-algebra in logarithmic CFT, Theor. Math. Phys. 148 (2006) 1210 [math/0512621] [INSPIRE].

[36] B.L. Feigin, A.M. Gainutdinov, A.M. Semikhatov and I.Y. Tipunin, Logarithmic extensions of minimal models: Characters and modular transformations, Nucl. Phys. B 757 (2006) 303 [hep-th/0606196] [INSPIRE].

[37] D. Adamovic and A. Milas, On the triplet vertex algebra W(p), Adv. Math. 217 (2008) 2664 [arXiv: 0707.1857] [INSPIRE].

[38] A. Tsuchiya and S. Wood, The tensor structure on the representation category of the $W_{p}$ triplet algebra, J. Phys. A 46 (2013) 445203 [arXiv:1201.0419] [InSPIRE].

[39] T. Creutzig, D. Ridout and S. Wood, Coset Constructions of Logarithmic $(1, p)$ Models, Lett. Math. Phys. 104 (2014) 553 [arXiv:1305.2665] [INSPIRE].

[40] M. Buican and T. Nishinaka, Argyres-Douglas Theories, the Macdonald Index and an RG Inequality, JHEP 02 (2016) 159 [arXiv: 1509.05402] [INSPIRE].

[41] M. Buican and T. Nishinaka, Argyres-Douglas theories, $S^{1}$ reductions and topological symmetries, J. Phys. A 49 (2016) 045401 [arXiv: 1505.06205] [InSPIRE].

[42] D. Gaiotto, L. Rastelli and S.S. Razamat, Bootstrapping the superconformal index with surface defects, JHEP 01 (2013) 022 [arXiv:1207.3577] [INSPIRE].

[43] J. Song, Superconformal indices of generalized Argyres-Douglas theories from $2 d$ TQFT, JHEP 02 (2016) 045 [arXiv: 1509.06730] [INSPIRE].

[44] L.F. Alday, M. Bullimore, M. Fluder and L. Hollands, Surface defects, the superconformal index and q-deformed Yang-Mills, JHEP 10 (2013) 018 [arXiv:1303.4460] [INSPIRE].

[45] M. Bullimore, M. Fluder, L. Hollands and P. Richmond, The superconformal index and an elliptic algebra of surface defects, JHEP 10 (2014) 062 [arXiv:1401.3379] [INSPIRE].

[46] H.-Y. Chen and H.-Y. Chen, Heterotic Surface Defects and Dualities from $2 d / 4 d$ Indices, JHEP 10 (2014) 004 [arXiv: 1407.4587] [INSPIRE].

[47] K. Maruyoshi and J. Yagi, Surface defects as transfer matrices, PTEP 2016 (2016) 113B01 [arXiv: 1606.01041] [INSPIRE].

[48] Y. Ito and Y. Yoshida, Superconformal index with surface defects for class $\mathcal{S}_{k}$, arXiv: 1606.01653 [INSPIRE].

[49] B. Nazzal and S.S. Razamat, Surface Defects in E-String Compactifications and the van Diejen Model, SIGMA 14 (2018) 036 [arXiv:1801.00960] [INSPIRE].

[50] S.S. Razamat, Flavored surface defects in $4 d \mathcal{N}=1$ SCFTs, arXiv:1808.09509 [INSPIRE].

[51] M. Buican, Z. Laczko and T. Nishinaka, Flowing from 16 to 32 Supercharges, JHEP 10 (2018) 175 [arXiv: 1807.02785] [INSPIRE].

[52] C. Beem, W. Peelaers, L. Rastelli and B.C. van Rees, Chiral algebras of class S, JHEP 05 (2015) 020 [arXiv: 1408.6522] [InSPIRE].

[53] V.A. Fateev and S.L. Lukyanov, The Models of Two-Dimensional Conformal Quantum Field Theory with Z(n) Symmetry, Int. J. Mod. Phys. A 3 (1988) 507 [INSPIRE].

[54] D. Altschuler, M. Bauer and H. Saleur, Level rank duality in nonunitary coset theories, J. Phys. A 23 (1990) L789 [INSPIRE]. 
[55] J. Song, D. Xie and W. Yan, Vertex operator algebras of Argyres-Douglas theories from M5-branes, JHEP 12 (2017) 123 [arXiv: 1706.01607] [INSPIRE].

[56] B.L. Feigin and A.M. Semikhatov, $W_{n}^{(2)}$ algebras, Nucl. Phys. B 698 (2004) 409 [math/0401164] [INSPIRE].

[57] T. Creutzig and D. Gaiotto, Vertex Algebras for S-duality, arXiv:1708.00875 [INSPIRE].

[58] D. Gaiotto and M. Rapčák, Vertex Algebras at the Corner, JHEP 01 (2019) 160 [arXiv:1703.00982] [INSPIRE].

[59] J. Song, Macdonald Index and Chiral Algebra, JHEP 08 (2017) 044 [arXiv:1612.08956] [INSPIRE].

[60] F. Bonetti, C. Meneghelli and L. Rastelli, VOAs labelled by complex reflection groups and 4d SCFTs, arXiv: 1810.03612 [INSPIRE].

[61] M. Fukuda, S. Nakamura, Y. Matsuo and R.-D. Zhu, $S H^{c}$ realization of minimal model CFT: triality, poset and Burge condition, JHEP 11 (2015) 168 [arXiv:1509.01000] [INSPIRE].

[62] V. Belavin, O. Foda and R. Santachiara, AGT, N-Burge partitions and $\mathcal{W}_{N}$ minimal models, JHEP 10 (2015) 073 [arXiv:1507.03540] [INSPIRE].

[63] D. Gaiotto and H.-C. Kim, Surface defects and instanton partition functions, JHEP 10 (2016) 012 [arXiv: 1412.2781] [INSPIRE].

[64] S. Gukov and E. Witten, Gauge Theory, Ramification, And The Geometric Langlands Program, hep-th/0612073 [INSPIRE].

[65] S. Gukov and E. Witten, Rigid Surface Operators, Adv. Theor. Math. Phys. 14 (2010) 87 [arXiv: 0804.1561] [INSPIRE].

[66] O. Chacaltana, J. Distler and Y. Tachikawa, Nilpotent orbits and codimension-two defects of $6 d N=(2,0)$ theories, Int. J. Mod. Phys. A 28 (2013) 1340006 [arXiv:1203.2930] [INSPIRE]. 